\title{
Systems of Hammerstein integral inclusions in Banach spaces with mixed monotone conditions
}

\section{Toktam Dinevari and Marlène Frigon ${ }^{*}$ (])}

\section{"Correspondence:}

frigon@dms.umontreal.ca Département de Mathématiques et de Statistique, Université de Montréal, C.P. 6128, succ. Centre-Ville, Montréal, Québec H3C 3J7, Canada

\section{Springer}

\begin{abstract}
In this paper, we establish the existence of solutions to systems of Hammerstein integral inclusions under mixed monotonicity type conditions. Existence of solutions to systems of differential inclusions with initial value condition or periodic boundary value condition are also obtained. Our results rely on fixed point theorems for multivalued weak contractions on complete metric spaces endowed with a graph.
\end{abstract}

MSC: Primary 45G15; secondary 45N05; 47H04; 47H09; 47H10; 34B15

Keywords: Hammerstein integral inclusion; differential inclusion; system of inclusions; multivalued map; contraction on graph; monotonicity; fixed point

\section{Introduction}

We consider the following system of Hammerstein integral inclusions:

$$
x_{i}(t) \in \int_{0}^{1} H_{i}\left(t, s, x_{1}(s), \ldots, x_{N}(s)\right) d s \quad \forall t \in[0,1], \forall i=1, \ldots, N .
$$

Here, $H_{i}:[0,1] \times[0,1] \times E_{1} \times \cdots \times E_{N} \rightarrow E_{i}$ are multivalued maps with nonempty values and $E_{i}$ are Banach spaces.

Existence results for this problem have been established by many authors when $N=1$. In particular, in [1-3], the existence of a solution was obtained by applying a set-valued Mönch type fixed point theorem for multivalued maps while it was deduced from a fixed point theorem for condensing multivalued maps in [4]. Lispschitz type conditions were imposed on $H_{1}$ in [5] and [6], where existence results were deduced from Nadler fixed point results for multivalued contractions and for $(\varepsilon, \lambda)$-uniformly locally contractive multivalued maps, respectively. In [7], Hong and Qiu considered the case where $E_{1}$ is endowed with a partial order. Assuming that $H_{1}$ is integrably bounded, increasing with respect to $x_{1}$ and assuming the existence of lower and upper solutions, he deduced the existence of a solution from a Mönch type result for multivalued maps in ordered Banach spaces.

In 2004, Ran and Reurings [8] established a fixed point result which is, in some sense, a combination of the Banach contraction principle and the Knaster-Tarski fixed point theorem in a partially ordered set. They considered a continuous, monotone, order preserving single-valued map $f$ defined on a complete metric space endowed with a partial ordering.

(c) 2015 Dinevari and Frigon. This article is distributed under the terms of the Creative Commons Attribution 4.0 International License (http://creativecommons.org/licenses/by/4.0/), which permits unrestricted use, distribution, and reproduction in any medium, provided you give appropriate credit to the original author(s) and the source, provide a link to the Creative Commons license, and indicate if changes were made. 
They assumed that $f$ satisfies a contraction condition not necessarily for all $x$ and $y$, but for those such that $x \leq y$. Their result was generalized by Nieto and Rodríguez-López [ 9 , 10] who weakened the continuity and the monotonicity assumptions. Later, Jachymski [11] presented an unification of the previous results by considering complete metric spaces endowed with a graph $G$. He introduced the notion of single-valued $G$-contraction for which he obtained fixed point results. Those results were extended to multivalued maps on complete metric space endowed with a graph in [12]. In particular, the notion of multivalued weak $G$-contraction was introduced. This notion was new even in the single-valued case.

Nieto and Rodríguez-López $[9,10]$ applied their fixed point results to periodic boundary value problems for a first order differential equation with a monotone right hand side satisfying a Lipschitz type condition. Assuming the existence of a lower solution (or an upper solution), they established the existence of a solution.

To the best of our knowledge, Opoicev [13, 14] was the first to establish fixed point results for single-valued operators defined on a Banach space ordered by a cone satisfying a mixed monotone condition. On the other hand, Guo and Lakshmikantham [15] obtained the existence of a coupled fixed point to a mixed monotone single-valued operator $T: D \times D \rightarrow E_{1}$ for $D \subset E_{1}$ and $E_{1}$ endowed with a partial order. Inspired by the results in $[9,10,15]$ and [8], Gnana Bhaskar and Lakshimikantham [16] established a coupled fixed point result for a mixed monotone operator satisfying a contraction-type condition. They applied their result to a periodic boundary value problem for a first order differential equation under a mixed monotone condition and under the existence of a coupled lower and upper solutions. In [17], fixed point results were obtained for multivalued mixed monotone operators. Those results were applied to establish the existence of a solution to an initial value problem for a system of differential inclusions with the right hand side satisfying a mixed monotone condition.

In this paper, we study the problem (1.1) with Banach spaces $E_{i}$ endowed with a partial order. We do not assume that the maps $H_{i}$ have closed or compact values. Our main existence result is established in Section 3 where we assume that the multivalued maps $H_{i}$ are nondecreasing or nonincreasing with respect to each variable $x_{j}$. The continuity type condition imposed on $H_{i}$ is weaker than the notion upper semi-continuity. In Sections 4 and 5 , we consider the particular cases where the maps $H_{i}$ are, respectively, nonincreasing and nondecreasing (upward or downward). This permits us to obtain existence results with a weaker continuity type condition. This condition can be made even weaker if the order on $E_{i}$ satisfies an extra condition. In Section 6, we consider the case where some maps $H_{i}$ can be nondecreasing and nonincreasing with respect to some $x_{j}$. Our existence results rely on a slight modification of the fixed point result for multivalued weak $G$-contractions obtained in [12] and which is presented in Section 2. The application of this fixed point result to our problem is very natural and appropriate. It is worth to point out that we do not use the theory of coupled fixed point results for mixed monotone operators. In Section 7, we present some examples of applications of our results to particular systems of integral equations and to a system of integral inclusions.

In what follows, for $E$ a Banach space, the space of continuous functions from $[0,1]$ to $E$ is denoted by $C([0,1], E)$ and endowed with the usual norm $\|u\|_{0}=\max \{\|u(t)\|: t \in$ $[0,1]\}$. For $p \in\left[0, \infty\left[\right.\right.$, we consider $L^{p}([0,1], E)$ the space of Bochner measurable functions $u:[0,1] \rightarrow E$ such that $\|u\|^{p}$ is Lebesgue integrable on $[0,1]$, and we denote $\|u\|_{p}=$ $\int_{0}^{1}\|u(s)\|^{p} d s$. For $p=\infty, L^{\infty}([0,1], E)$ is the space of Bochner measurable functions which 
are essentially bounded. This space is endowed with the usual norm $\|u\|_{\infty}$. We denote by $W^{1,1}([0,1], E)$ the Sobolev space of absolutely continuous functions $u:[0,1] \rightarrow E$ such that $u^{\prime} \in L^{1}([0,1], E)$.

\section{Preliminaries on multivalued $G$-contractions}

\subsection{Multivalued contractions on a metric space endowed with a graph}

We recall some notions and results concerning multivalued contractions on a metric space endowed with a graph obtained in [12].

Let $(X, d)$ be a complete metric space. We consider a directed graph $G$ such that the set of its vertices $V(G)=X$ and the set of its edges $E(G)$ has no parallel edges and contains $\Delta$ the diagonal in $X \times X$. We identify $G$ with the pair $(V(G), E(G))$.

For $x, y \in X$ and $m \in \mathbb{N},\left(x^{i}\right)_{i=0}^{m}$ is called an $m$-directed path from $x$ to $y$ if $x=x^{0}, y=x^{m}$, and $\left(x^{i-1}, x^{i}\right) \in E(G)$ for every $i=1, \ldots, m$. We denote

$$
\begin{aligned}
& {[x]_{G}^{m}=\{y \in X: \text { there is an } m \text {-directed path from } x \text { to } y\},} \\
& {[x]_{G}=\bigcup_{m \in \mathbb{N}}[x]_{G}^{m} .}
\end{aligned}
$$

Observe that $[x]_{G}^{1} \subset[x]_{G}^{2} \subset \cdots \subset[x]_{G}$, since $\Delta \subset E(G)$.

For $y \in[x]_{G}^{m}$ and $z \in[x]_{G}$, we define

$$
\begin{aligned}
& p_{m}(x, y)=\inf \left\{\sum_{i=1}^{m} d\left(x^{i-1}, x^{i}\right):\left(x^{i}\right)_{i=0}^{m} \text { is an } m \text {-directed path from } x \text { to } y\right\} \\
& p(x, z)=\inf \left\{\sum_{i=1}^{m} d\left(x^{i-1}, x^{i}\right):\left(x^{i}\right)_{i=0}^{m} \text { is an } m \text {-directed path from } x \text { to } z\right. \\
& \quad \text { for some } m \in \mathbb{N}\} .
\end{aligned}
$$

Notice that $p_{m}(x, y) \geq p_{k+m}(x, y)$ for all $k \in \mathbb{N}$, and

$$
p(x, z)=\inf \left\{p_{m}(x, z): m \in \mathbb{N} \text { such that } z \in[x]_{G}^{m}\right\},
$$

since $\Delta \subset E(G)$.

Definition 2.1 Let $F: X \rightarrow X$ be a multivalued mapping.

(1) Let $m \in \mathbb{N}$. We say that a sequence $\left\{x_{n}\right\}$ is a $G_{m}$-Picard trajectory from $x_{0}$ if $x_{n} \in\left[x_{n-1}\right]_{G}^{m} \cap F\left(x_{n-1}\right)$ for all $n \in \mathbb{N}$. We denote by $T_{m}\left(F, G, x_{0}\right)$, the set of all $G_{m}$-Picard trajectories from $x_{0}$.

(2) We say that a sequence $\left\{x_{n}\right\}$ is a G-Picard trajectory from $x_{0}$ if $x_{n} \in\left[x_{n-1}\right]_{G} \cap F\left(x_{n-1}\right)$ for all $n \in \mathbb{N}$. We denote by $T\left(F, G, x_{0}\right)$, the set of all $G$-Picard trajectories from $x_{0}$.

Definition 2.2 Let $F: X \rightarrow X$ be a multivalued mapping.

(1) Let $m \in \mathbb{N}$. We say that $F$ is $G_{m}$-Picard continuous from $x_{0}$ if the limit of any convergent sequence $\left\{x_{n}\right\} \in T_{m}\left(F, G, x_{0}\right)$ is a fixed point of $F$.

(2) We say that $F$ is G-Picard continuous from $x_{0}$ if the limit of any convergent sequence $\left\{x_{n}\right\} \in T\left(F, G, x_{0}\right)$ is a fixed point of $F$. 
We recall the notions of contractions with respect to $G$ introduced in [12].

Definition 2.3 Let $Y \subset X$ and $F: Y \rightarrow X$ a multivalued mapping with nonempty values.

(1) We say that $F$ is a $G$-contraction if there exists $\lambda \in] 0,1$ [ such that, for all $(x, y) \in E(G)$ and all $u \in F(x)$, there exists $v \in F(y)$ such that

$$
(u, v) \in E(G) \quad \text { and } \quad d(u, v) \leq \lambda d(x, y)
$$

(2) We say that $F$ is a weak $G$-contraction if there exists $\lambda \in] 0,1[$ such that, for all $x, y \in Y$ with $y \in[x]_{G}$, and all $u \in F(x)$, there exists $v \in F(y)$ such that

$$
v \in[u]_{G} \quad \text { and } \quad p(u, v) \leq \lambda p(x, y)
$$

It is easy to verify that a $G$-contraction is a weak $G$-contraction. An example of a weak $G$-contraction which is not a $G$-contraction is presented in [12].

\subsection{Fixed point results for $G$-contractions}

Here is a fixed point result for G-contraction established in [12].

Theorem 2.4 Let $F: X \rightarrow X$ be a multivalued G-contraction. Assume there exist $m \in \mathbb{N}$ and $x_{0} \in X$ such that $\left[x_{0}\right]_{G}^{m} \cap F\left(x_{0}\right) \neq \emptyset$ and $F$ is $G_{m}$-Picard continuous from $x_{0}$. Then there exists a $G_{m}$-Picard trajectory $\left\{x_{n}\right\}$ converging to $x$ a fixed point of $F$.

Remark 2.5 (1) Arguing as in [12], it can be shown that if $F: X \rightarrow X$ is a multivalued $G$-contraction, then there exists $\alpha \in] 0,1\left[\right.$ such that, for all $y \in[x]_{G}^{m}$ and all $u \in F(x)$, there exists $v \in F(y) \cap[u]_{G}^{m}$ such that $p_{m}(u, v) \leq \alpha p_{m}(x, y)$.

(2) The assumption of $G_{m}$-Picard continuity concerns

$$
\left\{\left\{x_{n}\right\} \in T_{m}\left(F, G, x_{0}\right):\left\{x_{n}\right\} \text { converges }\right\} .
$$

Looking at the proof of Theorem 2.4, one sees that it is sufficient for the limit of a sequence in the following set to be a fixed point of $F$ :

$$
\left\{\left\{x_{n}\right\} \in T_{m}\left(F, G, x_{0}\right): \sum_{n=1}^{\infty} p_{m}\left(x_{n-1}, x_{n}\right)<\infty\right\} .
$$

So, the assumption of $G_{m}$-Picard continuity can be weakened in Theorem 2.4.

In practice, one realizes that it happens that the pair $(u, v)$ in the definition of $G$-contraction satisfies also some other properties. Taking into account this fact and the previous remark, one can state a generalization of Theorem 2.4. Its proof is analogous to the proof of Theorem 2.4 and it is left to the reader.

Theorem 2.6 Let $F: X \rightarrow X$ be a multivalued map and, for every $(x, y) \in E(G)$, let a property $\mathcal{P}(x, y)$. Assume there exists $m \in \mathbb{N}$ such that the following conditions hold:

(i) there exist $x_{0}, x_{1} \in X$ such that $x_{1} \in\left[x_{0}\right]_{G}^{m} \cap F\left(x_{0}\right)$; 
(ii) there exists $\alpha \in] 0,1\left[\right.$ such that, for all $y \in[x]_{G}^{m}$ and all $u \in F(x)$, there exists $v \in F(y) \cap[u]_{G}^{m}$ such that

$$
p_{m}(u, v) \leq \alpha p_{m}(x, y) \text { and }(u, v) \text { satisfies the property } \mathcal{P}(x, y) \text {; }
$$

(iii) for any sequence $\left\{x_{n}\right\} \in T_{m}\left(F, G, x_{0}\right)$ (with $x_{0}, x_{1}$ given in (i)) such that $\left(x_{n+1}, x_{n+2}\right)$ satisfies property $\mathcal{P}\left(x_{n}, x_{n+1}\right)$ for every $n \in \mathbb{N}$ and

$$
\sum_{n=1}^{\infty} p_{m}\left(x_{n-1}, x_{n}\right)<\infty
$$

its limit is a fixed point of $F$.

Then there exists a $G_{m}$-Picard trajectory $\left\{x_{n}\right\}$ converging to $x$ a fixed point of $F$.

\subsection{Fixed point results for weak $G$-contractions}

Here is a fixed point result for weak G-contractions obtained in [12].

Theorem 2.7 Let $F: X \rightarrow X$ be a multivalued weak G-contraction. Assume there exists $x_{0} \in X$ such that $\left[x_{0}\right]_{G} \cap F\left(x_{0}\right) \neq \emptyset$ and $F$ is G-Picard continuous from $x_{0}$. Then there exists a G-Picard trajectory $\left\{x_{n}\right\}$ converging to $x$ a fixed point of $F$.

It is important to understand that if $F$ is a $G$-contraction then for $(x, y) \in E(G)$ and $u \in$ $F(x)$, there is $v \in F(y)$ such that there is an 1-directed path from $u$ to $v$ such that $d(u, v) \leq$ $\lambda d(x, y)$. On the other hand, if $F$ is a weak $G$-contraction, for $(x, y) \in E(G)$ and $u \in F(x)$, one cannot ensure that there is an appropriate element of $F(y)$ on an 1-directed path from $u$. Indeed, a suitable $v \in F(y)$ could be on an $N$-directed path from $u$ for some $N$ strictly bigger than 1. A particular case of weak $G$-contraction is when such $N$ is the same for all $(x, y) \in E(G)$. Also, it could happen in practice that $(u, v)$ satisfies some other properties. The proof of the following result is analogous to the proof of Theorem 2.7 (see the proof of Theorem 3.4 in [12]) and it is left to the reader.

Theorem 2.8 Let $F: X \rightarrow X$ be a multivalued map and $m, N \in \mathbb{N}$. Let $\mathcal{P}_{k}(x, y)$ be a property for every $y \in[x]_{G}^{k}$ for $k=m N^{n}$ with $n \in \mathbb{N} \cup\{0\}$. Assume the following assumptions:

(i) There exists $x_{0} \in X$ such that $\left[x_{0}\right]_{G}^{m} \cap F\left(x_{0}\right) \neq \emptyset$.

(ii) There exists $\alpha \in] 0,1\left[\right.$ such that, for every $x \in X, u \in F(x)$ and every $y \in[x]_{G}^{k}$ with $k=m N^{n}$ and $n \in \mathbb{N} \cup\{0\}$, there exists $v \in[u]_{G}^{k N} \cap F(y)$ such that

$$
p_{k N}(u, v) \leq \alpha p_{k}(x, y) \text { and }(u, v) \text { satisfies the property } \mathcal{P}_{k}(x, y)
$$

(iii) For any sequence $\left\{x_{n}\right\}$ (with $x_{0}$ given in (i)) such that $x_{n} \in F\left(x_{n-1}\right) \cap\left[x_{n-1}\right]_{G}^{m N^{n-1}}$, $\left(x_{n}, x_{n+1}\right)$ satisfies property $\mathcal{P}_{m N^{n-1}}\left(x_{n-1}, x_{n}\right)$ for every $n \in \mathbb{N}$, and

$$
\sum_{n=1}^{\infty} p_{m N^{n}}\left(x_{n}, x_{n+1}\right)<\infty
$$

its limit is a fixed point of $F$.

Then there exists a G-Picard trajectory $\left\{x_{n}\right\}$ converging to $x$ a fixed point of $F$. 


\section{Main results with mixed monotonicity type conditions}

\subsection{Systems of Hammerstein integral equations with mixed monotone conditions}

We consider the system of Hammerstein integral inclusions (1.1):

$$
x_{i}(t) \in \int_{0}^{1} H_{i}\left(t, s, x_{1}(s), \ldots, x_{N}(s)\right) d s \quad \forall t \in[0,1], \forall i=1, \ldots, N
$$

where $H_{i}:[0,1] \times[0,1] \times E_{1} \times \cdots \times E_{N} \rightarrow E_{i}$ are multivalued maps with nonempty values.

We assume that the Banach spaces $E_{i}$ are endowed with a partial order $\preceq$ satisfying:

(O) for $u, v \in L^{1}\left([0,1], E_{i}\right)$ such that $u(s) \preceq v(s)$ a.e. $s \in[0,1]$, one has

$$
\int_{0}^{1} u(s) d s \preceq \int_{0}^{1} v(s) d s
$$

In this section, we establish existence results in the case where the maps $H_{i}$ satisfy monotonicity type conditions with respect to each variable $x_{j}$. This could be nondecreasing type conditions with respect to some variables and nonincreasing type conditions with respect to the others.

We denote $E=E_{1} \times \cdots \times E_{N}$ the Banach space endowed with the norm $\left\|\left(x_{1}, \ldots, x_{N}\right)\right\|=$ $\left\|x_{1}\right\|+\cdots+\left\|x_{N}\right\|$, and $H:[0,1] \times[0,1] \times E \rightarrow E$ the multivalued map defined by $H(t, s, x)=$ $\left(H_{1}(t, s, x), \ldots, H_{N}(t, s, x)\right)$.

We define the multivalued map $\mathcal{H}: C([0,1], E) \rightarrow \mathcal{E}$ by

$$
\mathcal{H}(x)=\{w \in \mathcal{E}: w(t, s) \in H(t, s, x(s)) \text { a.e. } s \in[0,1], \forall t \in[0,1]\},
$$

where

$$
\begin{aligned}
\mathcal{E}= & \left\{w:[0,1] \times[0,1] \rightarrow E: w(t, \cdot) \in L^{1}([0,1], E) \forall t \in[0,1],\right. \\
& \text { and } \left.t \mapsto \int_{0}^{1} w(t, s) d s \text { is continuous }\right\} .
\end{aligned}
$$

We look for solutions of (1.1) which are fixed points of the multivalued map $F: C([0,1], E) \rightarrow C([0,1], E)$ defined by

$$
F(x)=\left\{u \in C([0,1], E): \text { there exists } w \in \mathcal{H}(x) \text { such that } u(t)=\int_{0}^{1} w(t, s) d s\right\} .
$$

Here is our first existence result.

Theorem 3.1 Let $H:[0,1] \times[0,1] \times E \rightarrow E$ be a multivalued map with nonempty values. Assume the following conditions hold:

(i) There exist $x_{0} \in C([0,1], E), w_{0} \in \mathcal{H}\left(x_{0}\right)$ and $\sigma_{0}:\{1, \ldots, N\} \rightarrow\{1,-1\}$ such that

$$
0 \preceq \sigma_{0}(i)\left(\int_{0}^{1} w_{0, i}(t, s) d s-x_{0, i}(t)\right) \quad \forall t \in[0,1], \forall i=1, \ldots, N .
$$

(ii) There exists $\phi:[0,1] \times[0,1] \rightarrow[0, \infty[$ such that

$$
\phi(t, \cdot) \in L^{1}([0,1]) \quad \forall t \in[0,1] \text { and } \sup _{t \in[0,1]}\|\phi(t, \cdot)\|_{1}<1
$$


and, for $j=1, \ldots, N$, there is a map $\sigma_{j}:\{1, \ldots, N\} \rightarrow\{1,-1\}$ such that, for every $x=\left(x_{1}, \ldots, x_{N}\right) \in C([0,1], E), w \in \mathcal{H}(x)$ and every $\hat{x}=\left(\hat{x}_{1}, \ldots, \hat{x}_{N}\right) \in C([0,1], E)$ such that $x_{i}=\hat{x}_{i}$ for $i \neq j$ and $x_{j}(s) \preceq \hat{x}_{j}(s)$ for all $s \in[0,1]\left(\operatorname{resp} . \hat{x}_{j}(s) \preceq x_{j}(s)\right.$ for all $s \in[0,1])$, there exists $\hat{w} \in \mathcal{H}(\hat{x})$ such that, a.e. $s \in[0,1]$ and all $t \in[0,1]$,

$$
\|w(t, s)-\hat{w}(t, s)\| \leq \phi(t, s)\|x-\hat{x}\|_{0}
$$

and

$$
\left.0 \preceq \sigma_{j}(i)\left(\hat{w}_{i}(t, s)-w_{i}(t, s)\right) \quad\left(\text { resp. } 0 \preceq \sigma_{j}(i)\left(w_{i}(t, s)-\hat{w}_{i}(t, s)\right)\right) \quad \forall i=1, \ldots, N\right) .
$$

(iii) For every $x, x_{n} \in C([0,1], E), w \in \mathcal{E}$ and $w_{n} \in \mathcal{H}\left(x_{n}\right)$, one has $w \in \mathcal{H}(x)$ if

(a) $x_{n} \rightarrow x$ and $x_{n}(t)=\int_{0}^{1} w_{n-1}(t, s) d s$ for all $t \in[0,1]$ and all $n \in \mathbb{N}$;

(b) $w_{n}(t, s) \rightarrow w(t, s)$ and $\left\|w_{n}(t, s)\right\| \leq M \phi(t, s)+\left\|w_{0}(t, s)\right\|$ a.e. $s \in[0,1]$, all $t \in[0,1]$, all $n \in \mathbb{N}$, and for some $M \geq 0$.

Then (1.1) has a solution.

Proof We consider on $C([0,1], E)$ the graph $G$ with $V(G)=C([0,1], E)$ and

$(\mathrm{E}(\mathrm{G}))$ one has $\left(\left(x_{1}, \ldots, x_{N}\right),\left(y_{1}, \ldots, y_{N}\right)\right) \in E(G)$ if and only if one of the following conditions holds:

(i) there exists $j \in\{1, \ldots, N\}$ such that, for all $s \in[0,1], x_{j}(s) \preceq y_{j}(s)$ and $x_{i}(s)=y_{i}(s)$ for all $i \neq j$;

(ii) there exists $j \in\{1, \ldots, N\}$ such that, for all $s \in[0,1], y_{j}(s) \preceq x_{j}(s)$ and $x_{i}(s)=y_{i}(s)$ for all $i \neq j$.

Let

$$
x_{1}(t)=\int_{0}^{1} w_{0}(t, s) d s \quad \forall t \in[0,1]
$$

where $w_{0}$ is given in Assumption (i). One has $x_{1} \in F\left(x_{0}\right)$. Observe that

$$
\left(x_{0,1}, x_{0,2}, \ldots, x_{0, N}\right),\left(x_{1,1}, x_{0,2}, \ldots, x_{0, N}\right), \ldots,\left(x_{1,1}, \ldots, x_{1, N}\right)
$$

is an $N$-directed path from $x_{0}$ to $x_{1}$. So, $x_{1} \in F\left(x_{0}\right) \cap\left[x_{0}\right]_{G}^{N}$.

We consider the following properties:

$\left(\mathrm{P}_{1}\right) \quad$ For $(x, \hat{x}) \in E(G)$, we say that $(u, \hat{u}) \in \mathcal{P}_{1}(x, \hat{x})$ if, for all $w \in \mathcal{H}(x)$ such that $u(t)=$ $\int_{0}^{1} w(t, s) d s$ for all $t \in[0,1]$, there exists $\hat{w} \in \mathcal{H}(\hat{x})$ such that

(a) $\hat{u}(t)=\int_{0}^{1} \hat{w}(t, s) d s$ for all $t \in[0,1]$;

(b) $\|w(t, s)-\hat{w}(t, s)\| \leq \phi(t, s)\|x-\hat{x}\|_{0}$ a.e. $s \in[0,1]$ and all $t \in[0,1]$.

$\left(\mathrm{P}_{N}\right) \quad$ For $y \in[x]_{G}^{N}$, we say that $(u, v) \in \mathcal{P}_{N}(x, y)$ if there exist $\left(x^{k}\right)_{k=0}^{N}$ and $\left(u^{k}\right)_{k=0}^{N} N$-directed paths from $x$ to $y$ and from $u$ to $v$, respectively, such that $\left(u^{k-1}, u^{k}\right) \in \mathcal{P}_{1}\left(x^{k-1}, x^{k}\right)$ for $k=1, \ldots, N$. Hence, for all $w^{0} \in \mathcal{H}(x)$ such that $u(t)=\int_{0}^{1} w^{0}(t, s) d s$, there exists $w^{k} \in \mathcal{H}\left(x^{k}\right)$ such that

$$
u^{k}(t)=\int_{0}^{1} w^{k}(t, s) d s
$$


and

$$
\begin{aligned}
& \left\|w^{0}(t, s)-w^{N}(t, s)\right\| \leq \sum_{k=1}^{N}\left\|w^{k-1}(t, s)-w^{k}(t, s)\right\| \leq \phi(t, s) p_{N}(x, y) \\
& \quad \text { a.e. } s \in[0,1], \forall t \in[0,1] .
\end{aligned}
$$

$\left(\mathrm{P}_{N^{n}}\right)$ For $n \geq 2$ and $y \in[x]_{G}^{N^{n}}$, the property $\mathcal{P}_{N^{n}}(x, y)$ is defined inductively. Hence, for $(u, v) \in \mathcal{P}_{N^{n}}(x, y)$, one has $v \in[u]_{G}^{N^{n+1}}$ and for all $w \in \mathcal{H}(x)$ such that $u(t)=$ $\int_{0}^{1} w(t, s) d s$, there exists $\hat{w} \in \mathcal{H}(y)$ such that $v(t)=\int_{0}^{1} \hat{w}(t, s) d s$ and

$$
\|w(t, s)-\hat{w}(t, s)\| \leq \phi(t, s) p_{N^{n}}(x, y) \quad \text { a.e. } s \in[0,1], \forall t \in[0,1] .
$$

We claim that $F$ satisfies Condition (ii) of Theorem 2.8 with

$$
\alpha \in] \sup _{t \in[0,1]}\|\phi(t, \cdot)\|_{1}, 1[.
$$

Indeed, let $(x, \hat{x}) \in E(G)$ and $u \in F(x)$. For $w \in \mathcal{H}(x)$ such that

$$
u(t)=\int_{0}^{1} w(t, s) d s \quad \forall t \in[0,1]
$$

let $\hat{w} \in \mathcal{H}(\hat{x})$ be ensured by Assumption (ii) and define

$$
\hat{u}(t)=\int_{0}^{1} \hat{w}(t, s) d s \quad \forall t \in[0,1] .
$$

Thus, by $(\mathrm{O})$, for $i=1, \ldots, N$,

$$
\begin{array}{ll}
u_{i}(t) \preceq \hat{u}_{i}(t) \quad \forall t \in[0,1], \quad \text { or } \\
\hat{u}_{i}(t) \preceq u_{i}(t) \quad \forall t \in[0,1] . &
\end{array}
$$

One has $\hat{u} \in[u]_{G}^{N}$, since

$$
\left(u_{1}, \ldots, u_{N}\right),\left(\hat{u}_{1}, u_{2}, \ldots, u_{N}\right), \ldots,\left(\hat{u}_{1}, \ldots, \hat{u}_{N}\right)
$$

is an $N$-directed path from $u$ to $\hat{u}$. Also, $(u, \hat{u}) \in \mathcal{P}_{1}(x, \hat{x})$ and

$$
\begin{aligned}
p_{N}(u, \hat{u}) \leq & \left\|\left(u_{1}, \ldots, u_{N}\right)-\left(\hat{u}_{1}, u_{2}, \ldots, u_{N}\right)\right\|_{0}+\cdots \\
& +\left\|\left(\hat{u}_{1}, \ldots, \hat{u}_{N-1}, u_{N}\right)-\left(\hat{u}_{1}, \ldots, \hat{u}_{N}\right)\right\|_{0} \\
= & \|u-\hat{u}\|_{0} \\
\leq & \lambda\|x-\hat{x}\|_{0}
\end{aligned}
$$

with

$$
\lambda=\sup _{t \in[0,1]}\|\phi(t, \cdot)\|_{1} .
$$


Fix $\varepsilon>0$ such that $\lambda(1+\varepsilon)<\alpha$. Let $y \in[x]_{G}^{N}$ and $u \in F(x)$. There exists $\left(x^{k}\right)_{k=0}^{N}$ an $N$-directed path from $x$ to $y$ such that

$$
\sum_{k=1}^{N}\left\|x^{k-1}-x^{k}\right\|_{0} \leq(1+\varepsilon) p_{N}(x, y)
$$

The previous argument ensures that, for $k=0, \ldots, N$, there exists $w^{k} \in \mathcal{H}\left(x^{k}\right)$ such that $u=u^{0}$,

$$
u^{k}(t)=\int_{0}^{1} w^{k}(t, s) d s \quad \forall t \in[0,1]
$$

and $\left(u^{k-1}, u^{k}\right) \in \mathcal{P}_{1}\left(x^{k-1}, x^{k}\right)$. So, for $v=u^{N}$, one has $(u, v) \in \mathcal{P}_{N}(x, y), v \in F(y) \cap[u]_{G}^{N^{2}}$, and

$$
\begin{aligned}
p_{N^{2}}(u, v) & \leq \sum_{k=1}^{N} p_{N}\left(u^{k-1}, u^{k}\right) \\
& \leq \lambda \sum_{k=1}^{N}\left\|x^{k-1}-x^{k}\right\| \\
& \leq \alpha p_{N}(x, y) .
\end{aligned}
$$

By induction on $n$, it can be shown that, for every $y \in[x]_{G}^{N^{n}}$ and every $u \in F(x)$, there exists $v \in F(y) \cap[u]_{G}^{N^{n+1}}$ such that $(u, v) \in \mathcal{P}_{N^{n}}(x, y)$ and

$$
p_{N^{n+1}}(u, v) \leq \alpha p_{N^{n}}(x, y)
$$

Finally, (iii) implies that Condition (iii) of Theorem 2.8 is satisfied. Indeed, let $\left\{x_{n}\right\}$ be such that $x_{n} \in F\left(x_{n-1}\right) \cap\left[x_{n-1}\right]_{G}^{N^{n}}$ and $\left(x_{n}, x_{n+1}\right) \in \mathcal{P}_{N^{n}}\left(x_{n-1}, x_{n}\right)$ for all $n \in \mathbb{N}$ and

$$
\sum_{n=1}^{\infty} p_{N^{n+1}}\left(x_{n}, x_{n+1}\right)<\infty
$$

Since

$$
\sum_{n=1}^{\infty}\left\|x_{n}-x_{n+1}\right\|_{0} \leq \sum_{n=1}^{\infty} p_{N^{n+1}}\left(x_{n}, x_{n+1}\right)
$$

$\left\{x_{n}\right\}$ is a Cauchy sequence converging to some $x \in C([0,1], E)$. Also, since $\left(x_{n}, x_{n+1}\right) \in$ $\mathcal{P}_{N^{n}}\left(x_{n-1}, x_{n}\right)$ for all $n \in \mathbb{N}$, there exists $w_{n} \in \mathcal{H}\left(x_{n}\right)$ such that $x_{n+1}=\int_{0}^{1} w_{n}(t, s) d s$ and

$$
\left\|w_{n-1}(t, s)-w_{n}(t, s)\right\| \leq \phi(t, s) p_{N^{n}}\left(x_{n-1}, x_{n}\right) \quad \text { a.e. } s \in[0,1], \forall t \in[0,1] .
$$

So,

$$
\left\|w_{n}(t, s)\right\| \leq\left\|w_{0}(t, s)\right\|+\phi(t, s) \sum_{n=1}^{\infty} p_{N^{n}}\left(x_{n-1}, x_{n}\right) \quad \text { a.e. } s \in[0,1], \forall t \in[0,1] .
$$


It follows from the Lebesgue dominated convergence theorem that there exists $w:[0,1] \times$ $[0,1] \rightarrow E$ such that $w(t, \cdot) \in L^{1}([0,1], E)$ and

$$
\left\|w(t, \cdot)-w_{n}(t, \cdot)\right\|_{1} \rightarrow 0 \quad \forall t \in[0,1]
$$

Also,

$$
x(t)=\lim _{n \rightarrow \infty} x_{n}(t)=\lim _{n \rightarrow \infty} \int_{0}^{1} w_{n}(t, s) d s=\int_{0}^{1} w(t, s) d s .
$$

So, $w \in \mathcal{E}$. Assumption (iii) implies that $w \in \mathcal{H}(x)$, and hence $x \in F(x)$.

Finally, Theorem 2.8 gives the conclusion.

Remark 3.2 A multivalued map $T: E_{j} \rightarrow E_{j}$ is said to be

- nondecreasing upward (resp. downward) if for every $x_{j} \in E_{j}, v \in T\left(x_{j}\right)$ and every $\hat{x}_{j} \in E_{j}$ such that $x_{j} \preceq \hat{x}_{j}\left(\right.$ resp. $\hat{x}_{j} \preceq x_{j}$ ), there exists $\hat{v} \in T\left(\hat{x}_{j}\right)$ such that $v \preceq \hat{v}$ (resp. $\hat{v} \preceq v$ );

- nonincreasing upward (resp. downward) if for every $x_{j} \in E_{j}, v \in T\left(x_{j}\right)$ and every $\hat{x}_{j} \in E_{j}$ such that $x_{j} \preceq \hat{x}_{j}\left(\right.$ resp. $\hat{x}_{j} \preceq x_{j}$ ), there exists $\hat{v} \in T\left(\hat{x}_{j}\right)$ such that $\hat{v} \preceq v$ (resp. $v \preceq \hat{v}$ );

- nondecreasing (resp. nonincreasing), it is nondecreasing (resp. nonincreasing) upward and downward.

Condition (ii) of the previous theorem implies that $H_{i}$ is nondecreasing (resp. nonincreasing) with respect to $x_{j}$ if $\sigma_{j}(i)=1\left(\operatorname{resp} . \sigma_{j}(i)=-1\right)$.

Remark 3.3 Observe that Condition (iii) in the previous theorem is satisfied if $x \mapsto$ $H(t, s, x)$ is upper semi-continuous (i.e. $\{x \in E: H(t, s, x) \cap B \neq \emptyset\}$ is closed for every closed $B \subset E)$ a.e. $s \in[0,1]$ and all $t \in[0,1]$.

\subsection{Some corollaries with mixed monotone conditions}

We present some corollaries of our main result. In the particular case where $H$ is singlevalued, we obtain the following result.

Corollary 3.4 Let $h:[0,1] \times[0,1] \times E \rightarrow E$ be a single-valued map. Assume the following conditions hold:

(i) For every $x \in C([0,1], E), h(t, \cdot x(\cdot)) \in L^{1}([0,1], E)$ for all $t \in[0,1]$, and $t \mapsto \int_{0}^{1} h(t, s, x(s)) d s$ is continuous.

(ii) There exist $\psi \in C([0,1], E)$ such that, for $i=1, \ldots, N$,

$$
\begin{array}{ll}
\psi_{i}(t) \preceq \int_{0}^{1} h_{i}(t, s, \psi(s)) d s \quad \forall t \in[0,1], \quad \text { or } \\
\int_{0}^{1} h_{i}(t, s, \psi(s)) d s \preceq \psi_{i}(t) \quad \forall t \in[0,1] .
\end{array}
$$

(iii) For $i, j=1, \ldots, N$, the map $x_{j} \mapsto h_{i}\left(t, s, x_{1}, \ldots, x_{j-1}, x_{j}, x_{j+1}, \ldots, x_{N}\right)$ is nondecreasing a.e. $s \in[0,1]$ and all $t \in[0,1]$, or it is nonincreasing a.e. $s \in[0,1]$ and all $t \in[0,1]$.

(iv) There exists $\phi:[0,1] \times[0,1] \rightarrow[0, \infty[$ such that

$$
\phi(t, \cdot) \in L^{1}([0,1]) \quad \forall t \in[0,1] \text { and } \sup _{t \in[0,1]}\|\phi(t, \cdot)\|_{1}<1
$$


and, for every $x, \hat{x} \in C([0,1], E)$ such that $x_{i}=\hat{x}_{i}$ for $i \neq j$ and $x_{j}(s) \preceq \hat{x}_{j}(s)$ for all $s \in[0,1]$, one has

$$
\|h(t, s, x(s))-h(t, s, \hat{x}(s))\| \leq \phi(t, s)\|x-\hat{x}\|_{0}
$$

(v) For every $x, x_{n} \in C([0,1], E)$, and $\hat{h}:[0,1] \times[0,1] \rightarrow E$, one has $\hat{h}(t, s)=h(t, s, x(s))$ a.e. $s \in[0,1]$ and all $t \in[0,1]$, if

(a) $x_{n} \rightarrow x$ and $x_{n+1}(t)=\int_{0}^{1} h\left(t, s, x_{n}(s)\right) d s$ for all $t \in[0,1]$ and all $n \in \mathbb{N}$;

(b) $h\left(t, s, x_{n}(s)\right) \rightarrow \hat{h}(t, s)$ and $\left\|h\left(t, s, x_{n}(s)\right)\right\| \leq M \phi(t, s)+\|h(t, s, \psi(s))\|$ a.e. $s \in[0,1]$, all $t \in[0,1]$, all $n \in \mathbb{N}$, and for some $M \geq 0$.

Then the following system of Hammerstein integral equations has a solution:

$$
x(t)=\int_{0}^{1} h(t, s, x(s)) d s \quad \forall t \in[0,1] .
$$

Here is an a corollary of Theorem 3.1 when $H$ can be written $H(t, s, x)=g(t, s) K(s, x)$ with $g$ single-valued.

Corollary 3.5 Let $K:[0,1] \times E \rightarrow E$ be a multivalued map with nonempty values and $g:[0,1] \times[0,1] \rightarrow\left[0, \infty\left[{ }^{N}\right.\right.$ a single-valued map. Let $p \in[1, \infty]$ and $q$ its conjugate. Assume the following conditions hold:

(i) For every $t \in[0,1], g(t, \cdot) \in L^{q}\left([0,1], \mathbb{R}^{N}\right)$ and $t \mapsto g(t, \cdot)$ is continuous.

(ii) There exist $\psi \in C([0,1], E)$ and $\mu \in L^{p}([0,1], E)$ such that $\mu(s) \in K(s, \psi(s))$ a.e.

$s \in[0,1]$, and for every $i=1, \ldots, N$

$$
\begin{array}{ll}
\psi_{i}(t) \preceq \int_{0}^{1} g_{i}(t, s) \mu_{i}(s) d s & \forall t \in[0,1], \quad \text { or } \\
\int_{0}^{1} g_{i}(t, s) \mu_{i}(s) d s \preceq \psi_{i}(t) \quad \forall t \in[0,1] .
\end{array}
$$

(iii) There exists $l \in L^{p}\left([0,1],\left[0, \infty\left[{ }^{N}\right)\right.\right.$ such that

$$
\alpha=\sup _{t \in[0,1]} \max \left\{\left\|l_{1}(\cdot) g_{1}(t, \cdot)\right\|_{1}, \ldots,\left\|l_{N}(\cdot) g_{N}(t, \cdot)\right\|_{1}\right\}<1,
$$

and for $j=1, \ldots, N$, there exists $\sigma_{j}:\{1, \ldots, N\} \rightarrow\{1,-1\}$ such that, for every $x \in C([0,1], E)$ and every $k \in L^{p}([0,1], E)$ such that $k(s) \in K(s, x(s))$ a.e. $s \in[0,1]$, one has, for every $\hat{x} \in C([0,1], E)$ such that $x_{i}=\hat{x}_{i}$ for $i \neq j$ and $x_{j}(s) \preceq \hat{x}_{j}(s)$ for all $s \in[0,1]\left(\operatorname{resp} . \hat{x}_{j}(s) \preceq x_{j}(s)\right.$ for all $\left.s \in[0,1]\right)$, there exists $\hat{k} \in L^{p}([0,1], E)$ such that, a.e. $s \in[0,1], \hat{k}(s) \in K(s, \hat{x}(s))$,

$$
\left\|\hat{k}_{i}(s)-k_{i}(s)\right\| \leq l_{i}(s)\left\|\hat{x}_{j}-x_{j}\right\|_{0}
$$

and

$$
\begin{aligned}
& 0 \preceq \sigma_{j}(i)\left(\hat{k}_{i}(s)-k_{i}(s)\right) \quad \forall i=1, \ldots, N \\
& \quad\left(\operatorname{resp} .0 \preceq \sigma_{j}(i)\left(k_{i}(s)-\hat{k}_{i}(s)\right) \quad \forall i=1, \ldots, N\right) .
\end{aligned}
$$


(iv) For every $x, x_{n} \in C([0,1], E)$ and for every $k, k_{n} \in L^{p}([0,1], E)$ such that $k_{n}(s) \in K\left(s, x_{n}(s)\right)$ a.e. $s \in[0,1]$, one has $k(s) \in K(s, x(s))$ a.e. $s \in[0,1]$ if

(a) $x_{n} \rightarrow x$ and $x_{n, i}(t)=\int_{0}^{1} g_{i}(t, s) k_{n-1, i}(s) d s$ for all $t \in[0,1], n \in \mathbb{N}$, and $i=1, \ldots, N$;

(b) $k_{n}(s) \rightarrow k(s)$ and $\left|k_{n, i}(s)\right| \leq M l_{i}(s)+\left|k_{0, i}(s)\right|$ a.e. $s \in[0,1]$, all $i=1, \ldots, N$, all $n \in \mathbb{N}$ and for some $M \geq 0$.

Then the following system has a solution:

$$
x_{i}(t) \in \int_{0}^{1} g_{i}(t, s) K_{i}(s, x(s)) d s \quad \forall t \in[0,1], i=1, \ldots, N .
$$

Now, we consider the initial value problem for a system of differential inclusions:

$$
\begin{aligned}
& x^{\prime}(t) \in K(t, x(t)) \quad \text { a.e. } t \in[0,1], \\
& x(0)=r .
\end{aligned}
$$

Corollary 3.6 Let $r \in E$ and $K:[0,1] \times E \rightarrow E$ a multivalued map with nonempty values. Assume the following conditions hold:

(i) There exist $\psi \in C([0,1], E), v \in L^{1}([0,1], E)$ such that $v(s) \in K(s, \psi(s))$ a.e. $s \in[0,1]$, and for all $i=1, \ldots, N$,

$$
\begin{array}{ll}
\psi_{i}(t) \preceq r_{i}+\int_{0}^{t} v_{i}(s) d s \quad \forall t \in[0,1], \quad \text { or } \\
r_{i}+\int_{0}^{t} v_{i}(s) d s \preceq \psi_{i}(t) \quad \forall t \in[0,1] .
\end{array}
$$

(ii) There exists $l \in L^{1}([0,1])$ such that $\|l\|_{1}<1$ and for $j=1, \ldots, N$, there exists $\sigma_{j}:\{1, \ldots, N\} \rightarrow\{1,-1\}$ such that, for every $x \in C([0,1], E)$ and every $k \in L^{1}([0,1], E)$ such that $k(s) \in K(s, x(s))$ a.e. $s \in[0,1]$, one has, for every $\hat{x} \in C([0,1], E)$ such that $x_{i}=\hat{x}_{i}$ for $i \neq j, x_{j}(s) \preceq \hat{x}_{j}(s)$ for all $s \in[0,1]$ (resp. $\hat{x}_{j}(s) \preceq x_{j}(s)$ for all $\left.s \in[0,1]\right)$, there exists $\hat{k} \in L^{1}([0,1], E)$ such that a.e. $s \in[0,1], \hat{k}(s) \in K(s, \hat{x}(s))$,

$$
\left\|\hat{k}_{i}(s)-k_{i}(s)\right\| \leq l(s)\|\hat{x}-x\|_{0},
$$

and

$$
\begin{aligned}
& 0 \preceq \sigma_{j}(i)\left(\hat{k}_{i}(s)-k_{i}(s)\right) \quad \forall i=1, \ldots, N \\
& \quad\left(\operatorname{resp} .0 \preceq \sigma_{j}(i)\left(k_{i}(s)-\hat{k}_{i}(s)\right) \quad \forall i=1, \ldots, N\right) .
\end{aligned}
$$

(iii) For every $x \in C([0,1], E), x_{n} \in W^{1,1}([0,1], E)$ and every $k \in L^{1}([0,1], E)$ such that

$$
\begin{aligned}
& x_{n}(0)=r, x_{n+1}^{\prime}(s) \in K\left(s, x_{n}(s)\right) \text { a.e. } s \in[0,1] \text {, one has } k(s) \in K(s, x(s)) \text { a.e. } s \in[0,1] \text { if } \\
& \left\|x_{n}-x\right\|_{0} \rightarrow 0, x_{n}^{\prime}(s) \rightarrow k(s) \text { a.e. } s \in[0,1] \text { and } \\
& \left\|x_{n}^{\prime}(s)\right\| \leq M l(s)+\|v(s)\| \text { a.e. } s \in[0,1], \forall n \in \mathbb{N} \text { for some } M \geq 0 .
\end{aligned}
$$

Then (3.6) has a solution. 
Proof Let $H:[0,1] \times[0,1] \times E \rightarrow E$ be given by

$$
H(t, s, x)=r+\chi_{[0, t]}(s) K(s, x)
$$

The conclusion follows from Theorem 3.1.

Remark 3.7 Observe that (i) of the previous corollary is satisfied if there exist $\sigma:\{1, \ldots, N\} \rightarrow\{1,-1\}, \psi \in W^{1,1}([0,1], E)$ and $v \in L^{1}([0,1], E)$ such that $v(s) \in K(s, \psi(s))$ a.e. $s \in[0,1]$, and, for all $i=1, \ldots, N$,

$$
\begin{aligned}
& \text { if } \sigma(i)=1, \quad \psi_{i}(0) \preceq r_{i}, \quad \psi_{i}^{\prime}(s) \preceq v_{i}(s) \quad \text { a.e. } s \in[0,1], \\
& \text { if } \sigma(i)=-1, \quad r_{i} \preceq \psi_{i}(0), \quad v_{i}(s) \preceq \psi_{i}^{\prime}(s) \quad \text { a.e. } s \in[0,1] .
\end{aligned}
$$

In the particular case where $N=1$ and $E_{1}=\mathbb{R}$, such a function $\psi$ is called a lower solution (resp. upper solution) of (3.6) if $\sigma(1)=1$ (resp. $\sigma(1)=-1)$.

We consider the periodic boundary value problem for a system of differential inclusions:

$$
\begin{aligned}
& x^{\prime}(t) \in K(t, x(t)) \quad \text { a.e. } t \in[0,1], \\
& x(0)=x(1) .
\end{aligned}
$$

Corollary 3.8 Let $K:[0,1] \times E \rightarrow$ E be a multivalued map with nonempty values. Assume the following conditions hold:

(i) There exist $\psi \in W^{1,1}([0,1], E)$ and $v \in L^{1}([0,1], E)$ such that $v(s) \in K(s, \psi(s))$ a.e. $s \in[0,1]$, and, for all $i=1, \ldots, N$,

$$
\begin{array}{lll}
\psi_{i}(0) \preceq \psi(1) & \text { and } \quad \psi_{i}^{\prime}(s) \preceq v_{i}(s) & \text { a.e. } s \in[0,1], \quad \text { or } \\
\psi_{i}(1) \preceq \psi(0) & \text { and } \quad v_{i}(s) \preceq \psi_{i}^{\prime}(s) & \text { a.e. } s \in[0,1] .
\end{array}
$$

(ii) There exist $0 \leq l<a$ and for $j=1, \ldots, N$, there exists $\sigma_{j}:\{1, \ldots, N\} \rightarrow\{1,-1\}$ such that, for every $x \in C([0,1], E)$ and every $k \in L^{1}([0,1], E)$ with $k(s) \in K(s, x(s))$ a.e. $s \in[0,1]$, one has, for every $\hat{x} \in C([0,1], E)$ such that $x_{i}=\hat{x}_{i}$ for all $i \neq j$ and $x_{j}(s) \preceq \hat{x}_{j}(s)$ for all $s \in[0,1]$ (resp. $\hat{x}_{j}(s) \preceq x_{j}(s)$ for all $\left.s \in[0,1]\right)$, there exists $\hat{k} \in L^{1}([0,1], E)$ such that a.e. $s \in[0,1], \hat{k}(s) \in K(s, \hat{x}(s))$,

$$
\left\|\hat{k}_{i}(s)+a \hat{x}_{i}(s)-k_{i}(s)-a x_{i}(s)\right\| \leq l\|\hat{x}-x\|_{0},
$$

and

$$
\begin{aligned}
& 0 \preceq \sigma_{j}(i)\left(\hat{k}_{i}(s)+a \hat{x}_{i}(s)-k_{i}(s)-a x_{i}(s)\right) \quad \forall i=1, \ldots, N \\
& \left(\operatorname{resp} .0 \preceq \sigma_{j}(i)\left(k_{i}(s)+a x_{i}(s)-\hat{k}_{i}(s)-a \hat{x}_{i}(s)\right) \quad \forall i=1, \ldots, N\right) .
\end{aligned}
$$

(iii) For every $x \in C([0,1], E), x_{n} \in W^{1,1}([0,1], E)$ and every $k \in L^{1}([0,1], E)$ such that $x_{n}(0)=x_{n}(1), x_{n+1}^{\prime}(s) \in K\left(s, x_{n}(s)\right)+a\left(x_{n}(s)-x_{n+1}(s)\right)$ a.e. $s \in[0,1]$, one has 


$$
\begin{gathered}
k(s) \in K(s, x(s)) \text { a.e. } s \in[0,1] \text { if }\left\|x_{n}-x\right\|_{0} \rightarrow 0, x_{n}^{\prime}(s) \rightarrow k(s) \text { a.e. } s \in[0,1] \text { and } \\
\left\|x_{n}^{\prime}(s)\right\| \leq M l+\|v(s)\| \quad \text { a.e. } s \in[0,1], \forall n \in \mathbb{N} \text { for some } M \geq 0 .
\end{gathered}
$$

Then (3.7) has a solution.

Proof Let $g:[0,1] \times[0,1] \rightarrow[0, \infty[$ be defined by

$$
g(t, s)= \begin{cases}\frac{e^{a(s-t+1)}}{e^{a}-1}, & \text { if } 0 \leq s \leq t \leq 1 \\ \frac{e^{a(s-t)}}{e^{a}-1}, & \text { if } 0 \leq t<s \leq 1\end{cases}
$$

A solution of (3.7) is a solution of

$$
x_{i}(t) \in \int_{0}^{1} g(t, s)\left(K_{i}(s, x(s))+a x_{i}(s)\right) d s \quad \forall t \in[0,1], i=1, \ldots, N .
$$

The conclusion follows from Corollary 3.5.

Remark 3.9 In the particular case where $N=1$ and $E_{1}=\mathbb{R}$, the function $\psi$ satisfying Condition (i) of Corollary 3.8 is called a lower solution (resp. upper solution) of (3.7) if $\sigma(1)=1($ resp. $\sigma(1)=-1)$.

Remark 3.10 In the previous corollary, we can replace $l<a$ by $l \in L^{1}([0,1])$ such that

$$
\sup _{t \in[0,1]} \int_{0}^{1} g(t, s) l(s) d s<1
$$

\section{Results with nonincreasing type conditions}

\subsection{Hammerstein integral equations with nonincreasing type conditions}

In the previous section, we established the existence of a solution to the system (1.1), where all $H_{i}$ satisfy monotonicity type conditions with respect to each variable $x_{j}$. In this section, we consider a particular case where $H$ satisfies a nonincreasing type condition. In this particular case, the continuity condition (see Condition (iii) of Theorem 3.1) can be weakened. Also, the use of weak $G$-contraction will not be necessary in the proof, since the associated operator will be a $G$-contraction.

Theorem 4.1 Let $H:[0,1] \times[0,1] \times E \rightarrow E$ be a multivalued map with nonempty values. Assume the following conditions hold:

(i) There exist $x_{0} \in C([0,1], E), w_{0} \in \mathcal{H}\left(x_{0}\right)$ and $\sigma:\{1, \ldots, N\} \rightarrow\{1,-1\}$ such that

$$
0 \preceq \sigma(i)\left(\int_{0}^{1} w_{0, i}(t, s) d s-x_{0, i}(t)\right) \quad \forall t \in[0,1], \forall i=1, \ldots, N
$$

(ii) There exists $\phi:[0,1] \times[0,1] \rightarrow[0, \infty[$ such that

$$
\phi(t, \cdot) \in L^{1}([0,1]) \quad \forall t \in[0,1] \text { and } \sup _{t \in[0,1]}\|\phi(t, \cdot)\|_{1}<1
$$


and, for every $x \in C([0,1], E), w \in \mathcal{H}(x)$ and every $\hat{x} \in C([0,1], E)$ such that

$$
\begin{aligned}
& 0 \preceq \sigma(i)\left(\hat{x}_{i}(s)-x_{i}(s)\right) \quad \forall s \in[0,1], \forall i=1, \ldots, N \\
& \left(\operatorname{resp} .0 \preceq \sigma(i)\left(x_{i}(s)-\hat{x}_{i}(s)\right) \quad \forall s \in[0,1], \forall i=1, \ldots, N\right),
\end{aligned}
$$

there exists $\hat{w} \in \mathcal{H}(\hat{x})$ such that, a.e. $s \in[0,1]$ and all $t \in[0,1]$,

$$
\|w(t, s)-\hat{w}(t, s)\| \leq \phi(t, s)\|x-\hat{x}\|_{0}
$$

and

$$
\begin{aligned}
& 0 \preceq \sigma(i)\left(w_{i}(t, s)-\hat{w}_{i}(t, s)\right) \quad \forall i=1, \ldots, N \\
& \quad\left(\operatorname{resp} .0 \preceq \sigma(i)\left(\hat{w}_{i}(t, s)-\hat{w}_{i}(t, s)\right) \quad \forall i=1, \ldots, N\right) .
\end{aligned}
$$

(iii) For every $x_{n} \in C([0,1], E)$ and $w_{n} \in \mathcal{H}\left(x_{n}\right)$ such that

$$
\sum_{n=1}^{\infty}\left\|x_{n}-x_{n+1}\right\|_{0}<\infty, \quad x_{n+1}(t)=\int_{0}^{1} w_{n}(t, s) d s
$$

and

$$
\begin{gathered}
\left\|w_{n}(t, s)-w_{n+1}(t, s)\right\| \leq \phi(t, s)\left\|x_{n}-x_{n+1}\right\| \\
\text { a.e. } s \in[0,1], \forall t \in[0,1], \forall n \in \mathbb{N}
\end{gathered}
$$

one has $w \in \mathcal{H}(x)$, where $\left\|x_{n}-x\right\|_{0} \rightarrow 0$ and $\left\|w_{n}-w\right\|_{1} \rightarrow 0$.

Then (1.1) has a solution.

Proof We consider on $C([0,1], E)$ the graph $G^{r}$ with $V\left(G^{r}\right)=C([0,1], E)$ and

$\left(\mathrm{E}\left(\mathrm{G}^{r}\right)\right)$ one has $(x, y) \in E\left(G^{r}\right)$ if and only if one of the following conditions hold:

(a) $0 \preceq \sigma(i)\left(\hat{x}_{i}(s)-x_{i}(s)\right)$ for all $s \in[0,1]$ and all $i=1, \ldots, N$;

(b) $0 \preceq \sigma(i)\left(x_{i}(s)-\hat{x}_{i}(s)\right)$ for all $s \in[0,1]$ and all $i=1, \ldots, N$.

Let

$$
x_{1}(t)=\int_{0}^{1} w_{0}(t, s) d s \quad \forall t \in[0,1] .
$$

It follows from (i) that $\left(x_{0}, x_{1}\right) \in E\left(G^{r}\right)$ and $x_{1} \in F\left(x_{0}\right)$.

We consider the following property:

$\left(\mathrm{P}^{r}\right)$ For $(x, \hat{x}) \in E\left(G^{r}\right)$, we say that $(u, \hat{u}) \in \mathcal{P}^{r}(x, \hat{x})$ if for all $w \in \mathcal{H}(x)$ such that $u(t)=$ $\int_{0}^{1} w(t, s) d s$, there exists $\hat{w} \in \mathcal{H}(\hat{x})$ such that

(a) $\hat{u}(t)=\int_{0}^{1} \hat{w}(t, s) d s$

(b) $\|w(t, s)-\hat{w}(t, s)\| \leq \phi(t, s)\|x-\hat{x}\|_{0}$ a.e. $s \in[0,1]$, and all $t \in[0,1]$.

Let $F$ be the associated multivalued map defined in (3.3). It follows from (ii) that $F$ satisfies Condition (ii) of Theorem 2.6 with $m=1$.

Finally, Condition (iii) of Theorem 2.6 follows from Assumption (iii). 
Remark 4.2 If $H$ satisfies (i) and (ii) of Theorem 3.1 with $\sigma_{0}, \ldots, \sigma_{N}$ such that

$$
\sigma_{0}(j) \sigma_{j}(i)=-\sigma_{0}(i) \quad \forall i, j=1, \ldots, N
$$

then $\sigma=\sigma_{0}$ satisfies (i) and (ii) of the previous theorem. Indeed, without loss of generality, let $x, \hat{x} \in C([0,1], E)$ be such that

$$
0 \preceq \sigma(i)\left(\hat{x}_{i}(s)-x_{i}(s)\right) \quad \forall s \in[0,1], \forall i=1, \ldots, N
$$

Let $w \in \mathcal{H}(x)$. By (ii) of Theorem 3.1, there exists $\hat{w}^{1} \in \mathcal{H}\left(\hat{x}_{1}, x_{2}, \ldots, x_{N}\right)$ such that

$$
\left\|w(t, s)-\hat{w}^{1}(t, s)\right\| \leq \phi(t, s)\left\|x_{1}-\hat{x}_{1}\right\|_{0},
$$

and

$$
\begin{array}{ll}
\text { if } \sigma(1)=1, & 0 \preceq \sigma_{1}(i)\left(\hat{w}_{i}^{1}(t, s)-w_{i}(t, s)\right) \quad \forall i=1, \ldots, N, \\
\text { if } \sigma(1)=-1, \quad 0 \preceq \sigma_{1}(i)\left(w_{i}(t, s)-\hat{w}_{i}^{1}(t, s)\right) \quad \forall i=1, \ldots, N .
\end{array}
$$

So, by (4.1),

$$
0 \preceq \sigma(i)\left(w_{i}(t, s)-\hat{w}_{i}^{1}(t, s)\right)=\sigma(1) \sigma_{1}(i)\left(\hat{w}_{i}^{1}(t, s)-w_{i}(t, s)\right) \quad \forall i=1, \ldots, N
$$

By the same argument, for $j=2, \ldots, N$, there exists $\hat{w}^{j} \in \mathcal{H}\left(\hat{x}_{1}, \ldots, \hat{x}_{j}, x_{j+1}, \ldots, x_{N}\right)$ such that

$$
\left\|\hat{w}^{j-1}(t, s)-\hat{w}^{j}(t, s)\right\| \leq \phi(t, s)\left\|x_{j}-\hat{x}_{j}\right\|_{0}
$$

and

$$
0 \preceq \sigma(i)\left(\hat{w}_{i}^{j-1}(t, s)-\hat{w}_{i}^{j}(t, s)\right)=\sigma(j) \sigma_{j}(i)\left(\hat{w}_{i}^{j}(t, s)-\hat{w}_{i}^{j-1}(t, s)\right) \quad \forall i=1, \ldots, N
$$

Therefore, $\hat{w}=\hat{w}^{N} \in \mathcal{H}(\hat{x})$ is such that

$$
\begin{aligned}
\|w(t, s)-\hat{w}(t, s)\| & \leq \sum_{j=1}^{N}\left\|\hat{w}^{j-1}(t, s)-\hat{w}^{j}(t, s)\right\| \leq \phi(t, s) \sum_{j=1}^{N}\left\|x_{j}-\hat{x}_{j}\right\|_{0} \\
& =\phi(t, s)\|x-\hat{x}\|_{0}
\end{aligned}
$$

and

$$
0 \preceq \sigma(i)\left(w_{i}(t, s)-\hat{w}_{i}(t, s)\right)=\sum_{j=1}^{N} \sigma(i)\left(\hat{w}_{i}^{j-1}(t, s)-\hat{w}_{i}^{j}(t, s)\right) \quad \forall i=1, \ldots, N
$$

\subsection{Some corollaries with nonincreasing type conditions}

As in the previous section, we obtain as corollaries existence results for systems of differential inclusions with initial value condition or periodic boundary value condition. 
Corollary 4.3 Let $r \in E$ and $K:[0,1] \times E \rightarrow E$ a multivalued map with nonempty values. Assume the following conditions hold:

(i) There exist $\sigma:\{1, \ldots, N\} \rightarrow\{1,-1\}, \psi \in C([0,1], E), v \in L^{1}([0,1], E)$ such that $v(s) \in K(s, \psi(s))$ a.e. $s \in[0,1]$, and

$$
\sigma(i)\left(\psi_{i}(t)-r_{i}-\int_{0}^{t} v_{i}(s) d s\right) \preceq 0 \quad \forall t \in[0,1], \forall i=1, \ldots, N
$$

(ii) There exists $l \in L^{1}([0,1])$ such that $\|l\|_{1}<1$ and for every $x \in C([0,1], E)$ and every $k \in L^{1}([0,1], E)$ such that $k(s) \in K(s, x(s))$ a.e. $s \in[0,1]$, one has, for every $\hat{x} \in C([0,1], E)$ such that

$$
\begin{aligned}
& 0 \preceq \sigma(i)\left(\hat{x}_{i}(s)-x_{i}(s)\right) \quad \forall s \in[0,1], \forall i=1, \ldots, N \\
& \left(\operatorname{resp} .0 \preceq \sigma(i)\left(x_{i}(s)-\hat{x}_{i}(s)\right) \quad \forall s \in[0,1], \forall i=1, \ldots, N\right),
\end{aligned}
$$

there exists $\hat{k} \in L^{1}([0,1], E)$ such that a.e. $s \in[0,1], \hat{k}(s) \in K(s, \hat{x}(s))$, $\|k(s)-\hat{k}(s)\| \leq l(s)\|\hat{x}-x\|_{0}$, and

$$
\begin{aligned}
& 0 \preceq \sigma(i)\left(k_{i}(s)-\hat{k}_{i}(s)\right) \quad \forall i=1, \ldots, N \\
& \left(\operatorname{resp} .0 \preceq \sigma(i)\left(\hat{k}_{i}(s)-k_{i}(s)\right) \quad \forall i=1, \ldots, N\right) .
\end{aligned}
$$

(iii) For every $x_{n} \in W^{1,1}([0,1], E)$ such that $x_{n}(0)=r, x_{n+1}^{\prime}(t) \in K\left(s, x_{n}(s)\right)$ a.e. $s \in[0,1]$,

$$
\sum_{n=1}^{\infty}\left\|x_{n}-x_{n+1}\right\|_{0}<\infty
$$

and

$$
\left\|x_{n}^{\prime}(s)-x_{n+1}^{\prime}(s)\right\| \leq l(s)\left\|x_{n-1}-x_{n}\right\|_{0} \quad \text { a.e. } s \in[0,1], \forall n \in \mathbb{N}
$$

one has $k(s) \in K(s, x(s))$ a.e. $s \in[0,1]$, where $\left\|x_{n}-x\right\|_{0} \rightarrow 0$ and $\left\|x_{n}^{\prime}-k\right\|_{1} \rightarrow 0$.

Then (3.6) has a solution.

Corollary 4.4 Let $K:[0,1] \times E \rightarrow E$ be a multivalued map with nonempty values. Assume the following conditions hold:

(i) There exist $\sigma:\{1, \ldots, N\} \rightarrow\{1,-1\}, \psi \in W^{1,1}([0,1], E)$ and $v \in L^{1}([0,1], E)$ such that $v(s) \in K(s, \psi(s))$ a.e. $s \in[0,1]$, and, for all $i=1, \ldots, N$,

$$
\sigma(i)\left(\psi_{i}(0)-\psi(1)\right) \preceq 0 \quad \text { and } \quad \sigma(i)\left(\psi_{i}^{\prime}(s)-v_{i}(s)\right) \preceq 0 \quad \text { a.e. } s \in[0,1]
$$

(ii) There exist $0 \leq l<a$ such that, for every $x \in C([0,1], E)$ and $k \in L^{1}([0,1], E)$ with $k(s) \in K(s, x(s))$ a.e. $s \in[0,1]$, one has, for every $\hat{x} \in C([0,1], E)$ such that

$$
\begin{aligned}
& 0 \preceq \sigma(i)\left(\hat{x}_{i}(s)-x_{i}(s)\right) \quad \forall s \in[0,1], \forall i=1, \ldots, N \\
& \left(\text { resp. } 0 \preceq \sigma(i)\left(x_{i}(s)-\hat{x}_{i}(s)\right) \quad \forall s \in[0,1], \forall i=1, \ldots, N\right),
\end{aligned}
$$


there exists $\hat{k} \in L^{1}([0,1], E)$ such that a.e. $s \in[0,1], \hat{k}(s) \in K(s, \hat{x}(s))$,

$$
\|k(s)+a x(s)-\hat{k}(s)-a \hat{x}(s)\| \leq l\|\hat{x}-x\|_{0},
$$

and

$$
\begin{aligned}
& 0 \preceq \sigma(i)\left(k_{i}(s)+a x_{i}(s)-\hat{k}_{i}(s)-a \hat{x}_{i}(s)\right) \quad \forall i=1, \ldots, N \\
& \left(\operatorname{resp} .0 \preceq \sigma(i)\left(\hat{k}_{i}(s)+a \hat{x}_{i}(s)-k_{i}(s)-a x_{i}(s)\right) \quad \forall i=1, \ldots, N\right) .
\end{aligned}
$$

(iii) For every $x_{n} \in W^{1,1}([0,1], E)$ such that $x_{n}(0)=x_{n}(1)$,

$$
\sum_{n=1}^{\infty}\left\|x_{n}-x_{n+1}\right\|_{0}<\infty, \quad x_{n+1}^{\prime}(s) \in K\left(s, x_{n}(s)\right)+a\left(x_{n}(s)-x_{n+1}(s)\right),
$$

and

$$
\begin{aligned}
& \left\|x_{n}^{\prime}(s)-x_{n+1}^{\prime}(s)+a x_{n-1}(s)-a x_{n}(s)\right\| \leq l\left\|x_{n-1}-x_{n}\right\|_{0} \\
& \quad \text { a.e. } s \in[0,1], \forall n \in \mathbb{N} ;
\end{aligned}
$$

one has $k(s) \in K(s, x(s))$ a.e. $s \in[0,1]$, where $\left\|x_{n}-x\right\|_{0} \rightarrow 0$ and $\left\|x_{n}^{\prime}-k\right\|_{1} \rightarrow 0$.

Then (3.7) has a solution.

Remark 4.5 In the particular case where $N=1, E_{1}=\mathbb{R}$ and $K$ is a continuous single-valued map, the previous corollary is due to Nieto and Rodríguez-López [10].

\section{Results with nondecreasing type conditions}

\subsection{Hammerstein integral equations with nondecreasing type conditions}

In the previous section, we considered the particular case where $H$ satisfies a nonincreasing type condition. In this section, we establish the existence of a solution to the system (1.1) in the particular case where $H$ satisfies a nondecreasing (upward or downward) type condition.

Theorem 5.1 Let $H:[0,1] \times[0,1] \times E \rightarrow E$ be a multivalued map with nonempty values. Assume the following conditions hold:

(i) There exist $x_{0} \in C([0,1], E), w_{0} \in \mathcal{H}\left(x_{0}\right)$ and $\sigma:\{1, \ldots, N\} \rightarrow\{1,-1\}$ such that

$$
0 \preceq \sigma(i)\left(\int_{0}^{1} w_{0, i}(t, s) d s-x_{0, i}(t)\right) \quad \forall t \in[0,1], \forall i=1, \ldots, N .
$$

(ii) There exists $\phi:[0,1] \times[0,1] \rightarrow[0, \infty[$ such that

$$
\phi(t, \cdot) \in L^{1}([0,1]) \quad \forall t \in[0,1] \text { and } \sup _{t \in[0,1]}\|\phi(t, \cdot)\|_{1}<1
$$

and, for every $x \in C([0,1], E), w \in \mathcal{H}(x)$ and every $\hat{x} \in C([0,1], E)$ such that

$$
0 \preceq \sigma(i)\left(\hat{x}_{i}(s)-x_{i}(s)\right) \quad \forall s \in[0,1], \forall i=1, \ldots, N
$$


there exists $\hat{w} \in \mathcal{H}(\hat{x})$ such that, a.e. $s \in[0,1]$ and all $t \in[0,1]$,

$$
\|w(t, s)-\hat{w}(t, s)\| \leq \phi(t, s)\|x-\hat{x}\|_{0}
$$

and

$$
0 \preceq \sigma(i)\left(\hat{w}_{i}(t, s)-w_{i}(t, s)\right) \quad \forall i=1, \ldots, N
$$

(iii) For every $x_{n} \in C([0,1], E)$ and $w_{n} \in \mathcal{H}\left(x_{n}\right)$ such that

$$
\sum_{n=1}^{\infty}\left\|x_{n}-x_{n+1}\right\|_{0}<\infty, \quad x_{n+1}(t)=\int_{0}^{1} w_{n}(t, s) d s
$$

and

$$
\begin{gathered}
\left\|w_{n}(t, s)-w_{n+1}(t, s)\right\| \leq \phi(t, s)\left\|x_{n}-x_{n+1}\right\| \\
\text { a.e. } s \in[0,1], \forall t \in[0,1], \forall n \in \mathbb{N},
\end{gathered}
$$

one has $w \in \mathcal{H}(x)$, where $\left\|x_{n}-x\right\|_{0} \rightarrow 0$ and $\left\|w_{n}-w\right\|_{1} \rightarrow 0$.

Then (1.1) has a solution.

Proof We consider on $C([0,1], E)$ the graph $G^{d}$ with $V\left(G^{d}\right)=C([0,1], E)$ and

$\left(\mathrm{E}\left(\mathrm{G}^{d}\right)\right)$ one has $(x, y) \in E\left(G^{d}\right)$ if and only if $0 \preceq \sigma(i)\left(\hat{x}_{i}(s)-x_{i}(s)\right)$ for all $s \in[0,1]$ and all $i=1, \ldots, N$.

We consider the following property:

$\left(\mathrm{P}^{d}\right)$ For $(x, \hat{x}) \in E\left(G^{d}\right)$, we say that $(u, \hat{u}) \in \mathcal{P}^{d}(x, \hat{x})$ if for all $w \in \mathcal{H}(x)$ such that $u(t)=$ $\int_{0}^{1} w(t, s) d s$, there exists $\hat{w} \in \mathcal{H}(\hat{x})$ such that

(a) $\hat{u}(t)=\int_{0}^{1} \hat{w}(t, s) d s$;

(b) $\|w(t, s)-\hat{w}(t, s)\| \leq \phi(t, s)\|x-\hat{x}\|_{0}$ a.e. $s \in[0,1]$, and all $t \in[0,1]$;

(c) $0 \preceq \sigma(i)\left(\hat{w}_{i}(t, s)-w_{i}(t, s)\right)$ for a.e. $s \in[0,1]$, all $t \in[0,1]$ and all $i=1, \ldots, N$.

It can be shown that the operator $F$ defined in (3.3) satisfies the assumptions of Theorem 2.6.

In the next result, we show that the continuity type condition (iii) of the previous result can be removed if one assumes that $H$ has closed values and if the order on the Banach spaces $E_{i}$ satisfies an additional property.

Theorem 5.2 Let $H:[0,1] \times[0,1] \times E \rightarrow E$ be a multivalued map with nonempty closed values. Assume that Assumptions (i) and (ii) of Theorem 5.1 are satisfied. In addition, assume that the following conditions hold:

(iii)' For every $i=1, \ldots, N$, and every sequence $\left\{a_{n}\right\}$ in $E_{i}$ such that $a_{n} \rightarrow a$ and $0 \preceq$ $\sigma(i)\left(a_{n+1}-a_{n}\right)$ for all $n \in \mathbb{N}$, one has $0 \preceq \sigma(i)\left(a-a_{n}\right)$ for all $n \in \mathbb{N}$.

Then (1.1) has a solution. 
Proof Let $G^{d}$ and $\mathcal{P}^{d}(x, \hat{x})$ be the graph and the property introduced in the proof of the previous theorem. As in its proof, it follows from (i) and (ii) that Assumptions (i) and (ii) of Theorem 2.6 are satisfied.

We claim that $F$ satisfies (iii) of Theorem 2.6 with $m=1$. Let $\left\{x_{n}\right\}$ in $T_{1}\left(F, G^{d}, x_{0}\right)$ be such that $\left(x_{n}, x_{n+1}\right) \in \mathcal{P}^{d}\left(x_{n-1}, x_{n}\right)$ for all $n \in \mathbb{N}$ and

$$
\sum_{n=1}^{\infty}\left\|x_{n-1}-x_{n}\right\|_{0}<\infty .
$$

This a Cauchy sequence converging to some $x \in C([0,1], E)$. Since $\left(x_{n}, x_{n+1}\right) \in \mathcal{P}^{d}\left(x_{n-1}, x_{n}\right)$ for all $n \in \mathbb{N}$, there exists $w_{n} \in \mathcal{H}\left(x_{n}\right)$ such that $x_{n+1}=\int_{0}^{1} w_{n}(t, s) d s$,

$$
\left\|w_{n-1}(t, s)-w_{n}(t, s)\right\| \leq \phi(t, s)\left\|x_{n-1}-x_{n}\right\|_{0},
$$

and

$$
0 \preceq \sigma(i)\left(w_{n, i}(t, s)-w_{n-1, i}(t, s)\right) \quad \text { a.e. } s \in[0,1], \forall t \in[0,1], \forall i=1, \ldots, N, \forall n \in \mathbb{N} .
$$

Also,

$$
\left\|w_{n}(t, s)\right\| \leq\left\|w_{0}(t, s)\right\|+\phi(t, s) \sum_{n=1}^{\infty}\left\|x_{n-1}-x_{n}\right\|_{0} \quad \text { a.e. } s \in[0,1], \forall t \in[0,1] .
$$

It follows from the Lebesgue dominated convergence theorem that there exists $w:[0,1] \times$ $[0,1] \rightarrow E$ such that $w(t, \cdot) \in L^{1}([0,1], E)$ and

$$
\left\|w(t, \cdot)-w_{n}(t, \cdot)\right\|_{1} \rightarrow 0 \quad \forall t \in[0,1]
$$

Moreover,

$$
x(t)=\lim _{n \rightarrow \infty} x_{n}(t)=\lim _{n \rightarrow \infty} \int_{0}^{1} w_{n}(t, s) d s=\int_{0}^{1} w(t, s) d s \quad \forall t \in[0,1] .
$$

So, $w \in \mathcal{E}$. Also, by (iii) $)^{\prime}, 0 \preceq \sigma(i)\left(x_{i}(t)-x_{n, i}\right)(t)$ for all $t \in[0,1]$ and all $i=1, \ldots, N$. It follows from (ii) that, for every $n \in \mathbb{N}$, there exists $\hat{w}_{n} \in \mathcal{H}(x)$ such that

$$
\left\|w_{n}(t, s)-\hat{w}_{n}(t, s)\right\| \leq \phi(t, s)\left\|x_{n}-x\right\|_{0},
$$

and

$$
0 \preceq \sigma(i)\left(\hat{w}_{n}(t, s)-w_{n}(t, s)\right) \text { a.e. } s \in[0,1], \forall t \in[0,1], \forall i=1, \ldots, N \text {. }
$$

Therefore,

$$
\hat{w}_{n}(t, s) \rightarrow w(t, s) \quad \text { a.e. } s \in[0,1], \forall t \in[0,1] .
$$

The fact that $H$ has closed values implies that $w \in \mathcal{H}(x)$, and hence $x \in F(x)$.

Finally, Theorem 2.6 gives the conclusion. 
Remark 5.3 Assumption (ii) of Theorems 5.1 and 5.2 can be weakened by restricting the condition to $x \in C([0,1], E)$ such that $0 \preceq \sigma(i)\left(x_{i}(s)-x_{0, i}(s)\right)$ for all $s \in[0,1]$ and $i=1, \ldots, n$. So, Theorem 5.1 and 5.2 hold with (ii) replaced by

(ii) $)^{\prime}$ There exists $\phi:[0,1] \times[0,1] \rightarrow[0, \infty[$ such that

$$
\phi(t, \cdot) \in L^{1}([0,1]) \quad \forall t \in[0,1] \quad \text { and } \sup _{t \in[0,1]}\|\phi(t, \cdot)\|_{1}<1
$$

and, for every $x \in C([0,1], E), w \in \mathcal{H}(x)$ and every $\hat{x} \in C([0,1], E)$ such that

$$
\begin{aligned}
& 0 \preceq \sigma(i)\left(x_{i}(s)-x_{0, i}(s)\right) \quad \text { and } \\
& 0 \preceq \sigma(i)\left(\hat{x}_{i}(s)-x_{i}(s)\right) \quad \forall s \in[0,1], \forall i=1, \ldots, N,
\end{aligned}
$$

there exists $\hat{w} \in \mathcal{H}(\hat{x})$ such that, a.e. $s \in[0,1]$ and all $t \in[0,1]$,

$$
\|w(t, s)-\hat{w}(t, s)\| \leq \phi(t, s)\|x-\hat{x}\|_{0}
$$

and

$$
0 \preceq \sigma(i)\left(\hat{w}_{i}(t, s)-w_{i}(t, s)\right) \quad \forall i=1, \ldots, N .
$$

\subsection{Some corollaries with nondecreasing type conditions}

We state existence results for systems of differential inclusions with initial value condition or periodic boundary value condition which follow directly from the previous theorem.

Corollary 5.4 Let $r \in E$ and $K:[0,1] \times E \rightarrow E$ a multivalued map with nonempty closed values. Assume the following conditions hold:

(i) There exist $\sigma:\{1, \ldots, N\} \rightarrow\{1,-1\}, \psi \in C([0,1], E), v \in L^{1}([0,1], E)$ such that $v(s) \in K(s, \psi(s))$ a.e. $s \in[0,1]$, and

$$
\sigma(i)\left(\psi_{i}(t)-r_{i}-\int_{0}^{t} v_{i}(s) d s\right) \preceq 0 \quad \forall t \in[0,1], \forall i=1, \ldots, N
$$

(ii) There exists $l \in L^{1}([0,1])$ such that $\|l\|_{1}<1$ and for every $x \in C([0,1], E)$ and every $k \in L^{1}([0,1], E)$ such that $k(s) \in K(s, x(s))$ a.e. $s \in[0,1]$, for every $\hat{x} \in C([0,1], E)$ such that

$$
0 \preceq \sigma(i)\left(\hat{x}_{i}(s)-x_{i}(s)\right) \quad \forall s \in[0,1], \forall i=1, \ldots, N
$$

there exists $\hat{k} \in L^{1}([0,1], E)$ such that a.e. $s \in[0,1], \hat{k}(s) \in K(s, \hat{x}(s))$,

$$
\|k(s)-\hat{k}(s)\| \leq l(s)\|\hat{x}-x\|_{0}
$$

and

$$
0 \preceq \sigma(i)\left(\hat{k}_{i}(s)-k_{i}(s)\right) \quad \forall i=1, \ldots, N
$$


(iii) For every $i=1, \ldots, N$, and every sequence $\left\{a_{n}\right\}$ in $E_{i}$ such that $a_{n} \rightarrow a$ and $0 \preceq \sigma(i)\left(a_{n+1}-a_{n}\right)$ for all $n \in \mathbb{N}$, one has $0 \preceq \sigma(i)\left(a-a_{n}\right)$ for all $n \in \mathbb{N}$.

Then (3.6) has a solution.

Corollary 5.5 Let $K:[0,1] \times E \rightarrow E$ be a multivalued map with nonempty closed values. Assume the following conditions hold:

(i) There exist $\sigma:\{1, \ldots, N\} \rightarrow\{1,-1\}, \psi \in W^{1,1}([0,1], E)$ and $v \in L^{1}([0,1], E)$ such that $v(s) \in K(s, \psi(s))$ a.e. $s \in[0,1]$, and, for all $i=1, \ldots, N$,

$$
\sigma(i)\left(\psi_{i}(0)-\psi(1)\right) \preceq 0 \quad \text { and } \quad \sigma(i)\left(\psi_{i}^{\prime}(s)-v_{i}(s)\right) \preceq 0 \quad \text { a.e. } s \in[0,1]
$$

(ii) There exist $0 \leq l<a$ such that, for every $k \in L^{1}([0,1], E)$ with $k(s) \in K(s, x(s))$ a.e. $s \in[0,1]$, one has, for every $\hat{x} \in C([0,1], E)$ such that

$$
0 \preceq \sigma(i)\left(\hat{x}_{i}(s)-x_{i}(s)\right) \quad \forall s \in[0,1], \forall i=1, \ldots, N
$$

there exists $\hat{k} \in L^{1}([0,1], E)$ such that a.e. $s \in[0,1], \hat{k}(s) \in K(s, \hat{x}(s))$,

$$
\|k(s)+a x(s)-\hat{k}(s)-a \hat{x}(s)\| \leq l\|\hat{x}-x\|_{0},
$$

and

$$
0 \preceq \sigma(i)\left(\hat{k}_{i}(s)+a \hat{x}_{i}(s)-k_{i}(s)-a x_{i}(s)\right) \quad \forall i=1, \ldots, N
$$

(iii) For every $i=1, \ldots, N$, and every sequence $\left\{a_{n}\right\}$ in $E_{i}$ such that $a_{n} \rightarrow a$ and $0 \preceq \sigma(i)\left(a_{n+1}-a_{n}\right)$ for all $n \in \mathbb{N}$, one has $0 \preceq \sigma(i)\left(a-a_{n}\right)$ for all $n \in \mathbb{N}$.

Then (3.7) has a solution.

Remark 5.6 In the particular case where $N=1, E_{1}=\mathbb{R}$ and $K$ is a continuous singlevalued map, the previous corollary is due to Nieto and Rodríguez-López [9].

\section{Other results}

It could happen that a map $H_{i}$ satisfies at the same time a nonincreasing type condition and a nondecreasing type condition with respect to some variables. For instance, for $x, \hat{x}, \tilde{x} \in E$ such that $\tilde{x}_{1} \preceq x_{1} \preceq \hat{x}_{1}$ and $x_{j}=\hat{x}_{j}=\tilde{x}_{j}$ for $j \neq 1$, and for $w_{i} \in H_{i}(t, s, x)$, there could exist $\hat{w}_{i} \in H_{i}(t, s, \hat{x}), \tilde{w}_{i} \in H_{i}(t, s, \tilde{x})$ such that $w_{i} \preceq \hat{w}_{i}$ and $w_{i} \preceq \tilde{w}_{i}$. If such $H_{i}$ is single-valued, that would imply that $H_{i}$ is constant with respect to $x_{1}$. However, in the multivalued case, such a $H_{i}$ does not need to be constant with respect to $x_{1}$. In this section, we consider the system (1.1) in which some of the maps $H_{i}$ satisfy this type of property. In some sense, we combine assumptions used in Sections 3 and 5.

In order to simplify the notation, we write $E=E_{*} \times E_{* *}$ with

$$
E_{*}=\prod_{i=1}^{N_{*}} E_{i} \quad \text { and } \quad E_{* *}=\prod_{i=1}^{N_{* *}} E_{i+N_{*}},
$$

where $N=N_{*}+N_{* *}$. We write $(x, y) \in E$ with $x \in E_{*}$ and $y \in E_{* *}$. 
Theorem 6.1 Let $H:[0,1] \times[0,1] \times E \rightarrow E$ be a multivalued map with nonempty values. Assume the following conditions hold:

(i) There exist $\left(x_{0}, y_{0}\right) \in C([0,1], E),\left(v_{0}, w_{0}\right) \in \mathcal{H}\left(x_{0}, y_{0}\right), \sigma_{*}:\left\{1, \ldots, N_{*}\right\} \rightarrow\{1,-1\}$ and $\sigma_{* *}:\left\{1, \ldots, N_{* *}\right\} \rightarrow\{1,-1\}$ such that

$$
\begin{aligned}
& 0 \preceq \sigma_{*}(i)\left(\int_{0}^{1} v_{0, i}(t, s) d s-x_{0, i}(t)\right) \quad \forall t \in[0,1], \forall i=1, \ldots, N_{*} ; \\
& 0 \preceq \sigma_{* *}(i)\left(\int_{0}^{1} w_{0, i}(t, s) d s-y_{0, i}(t)\right) \quad \forall t \in[0,1], \forall i=1, \ldots, N_{* *} .
\end{aligned}
$$

(ii) There exists $\phi:[0,1] \times[0,1] \rightarrow[0, \infty[$ such that

$$
\phi(t, \cdot) \in L^{1}([0,1]) \quad \forall t \in[0,1] \text { and } \sup _{t \in[0,1]}\|\phi(t, \cdot)\|_{1}<1
$$

and, for $j=0,1, \ldots, N_{* *}$, there is a map $\sigma_{j}:\left\{1, \ldots, N_{* *}\right\} \rightarrow\{1,-1\}$ such that, for every $(x, y) \in C([0,1], E),(v, w) \in \mathcal{H}(x, y)$, one has

(a) for every $\hat{x} \in C\left([0,1], E_{*}\right)$ such that

$$
0 \preceq \sigma_{*}(i)\left(\hat{x}_{i}(s)-x_{i}(s)\right) \quad \forall s \in[0,1], \forall i=1, \ldots, N_{*}
$$

there exists $(\hat{v}, \hat{w}) \in \mathcal{H}(\hat{x}, y)$ such that, a.e. $s \in[0,1]$ and all $t \in[0,1]$,

$$
\|(v(t, s), w(t, s))-(\hat{v}(t, s), \hat{w}(t, s))\| \leq \phi(t, s)\|x-\hat{x}\|_{0},
$$

and

$$
\begin{aligned}
& 0 \preceq \sigma_{*}(i)\left(\hat{v}_{i}(t, s)-v_{i}(t, s)\right) \quad \forall i=1, \ldots, N_{*}, \\
& 0 \preceq \sigma_{0}(i)\left(\hat{w}_{i}(t, s)-w_{i}(t, s)\right) \quad \forall i=1, \ldots, N_{* *} ;
\end{aligned}
$$

(b) for $j=1, \ldots, N_{* *}$, and every $\hat{y} \in C\left([0,1], E_{* *}\right)$ such that $y_{i}=\hat{y}_{i}$ for $i \neq j$ and $y_{j}(s) \preceq \hat{y}_{j}(s)$ for all $s \in[0,1]$ (resp. $\hat{y}_{j}(s) \preceq y_{j}(s)$ for all $\left.s \in[0,1]\right)$, there exists $(\hat{v}, \hat{w}) \in \mathcal{H}(x, \hat{y})$ such that, a.e. $s \in[0,1]$ and all $t \in[0,1]$,

$$
\|(v(t, s), w(t, s))-(\hat{v}(t, s), \hat{w}(t, s))\| \leq \phi(t, s)\|y-\hat{y}\|_{0},
$$

and

$$
\begin{aligned}
& 0 \preceq \sigma_{*}(i)\left(\hat{v}_{i}(t, s)-v_{i}(t, s)\right) \quad \forall i=1, \ldots, N_{*} \\
& 0 \preceq \sigma_{j}(i)\left(\hat{w}_{i}(t, s)-w_{i}(t, s)\right) \quad \forall i=1, \ldots, N_{* *} \\
& \left.\quad\left(\operatorname{resp} .0 \preceq \sigma_{j}(i)\left(w_{i}(t, s)-\hat{w}_{i}(t, s)\right)\right) \quad \forall i=1, \ldots, N_{* *}\right) .
\end{aligned}
$$

(iii) For every $i=1, \ldots, N_{*}$, and every sequence $\left\{a_{n}\right\}$ in $E_{i}$ such that $a_{n} \rightarrow a$ and $0 \preceq \sigma_{*}(i)\left(a_{n+1}-a_{n}\right)$ for all $n \in \mathbb{N}$, one has $0 \preceq \sigma_{*}(i)\left(a-a_{n}\right)$ for all $n \in \mathbb{N}$.

(iv) For every $(x, y) \in C([0,1], E), y_{n} \in C\left([0,1], E_{* *}\right),(v, w) \in \mathcal{E}$ and $\left(v_{n}, w_{n}\right) \in \mathcal{H}\left(x, y_{n}\right)$, one has $(v, w) \in \mathcal{H}(x, y)$ if 
(a) $y_{n} \rightarrow y$ and $y_{n}(t)=\int_{0}^{1} w_{n-1}(t, s) d s$ for all $t \in[0,1]$ and all $n \in \mathbb{N}$;

(b) $\left(v_{n}(t, s), w_{n}(t, s)\right) \rightarrow(v(t, s), w(t, s))$ and

$$
\begin{aligned}
& \left\|\left(v_{n}(t, s), w_{n}(t, s)\right)\right\| \leq M \phi(t, s)+\left\|\left(v_{0}(t, s), w_{0}(t, s)\right)\right\| \\
& \quad \text { a.e. } s \in[0,1], \forall t \in[0,1], \forall n \in \mathbb{N} \text {, and for some } M \geq 0 .
\end{aligned}
$$

Then (1.1) has a solution.

Proof We consider on $C([0,1], E)$ the graph $G^{*}$ with $V\left(G^{*}\right)=C([0,1], E)$ and

$\left(E\left(G^{*}\right)\right)$ one has $((x, y),(\hat{x}, \hat{y})) \in E\left(G^{*}\right)$ if and only if one of the following conditions holds:

(i) $y=\hat{y}$ and $0 \preceq \sigma_{*}(i)\left(\hat{x}_{i}(s)-x_{i}(s)\right)$ for all $s \in[0,1]$ and all $i=1, \ldots, N_{*}$;

(ii) $x=\hat{x}$ and there exists $j \in\left\{1, \ldots, N_{* *}\right\}$ such that, for all $s \in[0,1], y_{j}(s) \preceq \hat{y}_{j}(s)$ and $y_{i}(s)=\hat{y}_{i}(s)$ for all $i \neq j$;

(iii) $x=\hat{x}$ and there exists $j \in\left\{1, \ldots, N_{* *}\right\}$ such that, for all $s \in[0,1], \hat{y}_{j}(s) \preceq y_{j}(s)$ and $y_{i}(s)=\hat{y}_{i}(s)$ for all $i \neq j$.

We consider the following properties with $m=N_{* *}+1$ :

$\left(\mathrm{P}_{1}\right) \quad$ For $((x, y),(\hat{x}, \hat{y})) \in E\left(G^{*}\right)$, we say that $((u, \mu),(\hat{u}, \hat{\mu})) \in \mathcal{P}_{1}^{*}((x, y),(\hat{x}, \hat{y}))$ if for all $(v, w) \in$ $\mathcal{H}(x, y)$ such that

$$
(u(t), \mu(t))=\left(\int_{0}^{1} v(t, s) d s, \int_{0}^{1} w(t, s) d s\right) \quad \forall t \in[0,1]
$$

there exists $(\hat{v}, \hat{w}) \in \mathcal{H}(\hat{x}, \hat{y})$ such that

(a) one has

$$
(\hat{u}(t), \hat{\mu}(t))=\left(\int_{0}^{1} \hat{v}(t, s) d s, \int_{0}^{1} \hat{w}(t, s) d s\right) \quad \forall t \in[0,1]
$$

(b) $\|(v(t, s), w(t, s))-(\hat{v}(t, s), \hat{w}(t, s))\| \leq \phi(t, s)\|(x, y)-(\hat{x}, \hat{y})\|_{0}$ a.e. $s \in[0,1]$ and all $t \in[0,1]$

(c) $0 \preceq \sigma_{*}(i)\left(\hat{v}_{i}(t, s)-v_{i}(t, s)\right)$ a.e. $s \in[0,1]$, all $t \in[0,1]$, and all $i=1, \ldots, N_{*}$.

$\left(\mathrm{P}_{m}\right) \quad$ For $(\hat{x}, \hat{y}) \in[(x, y)]_{G}^{m}$, we say that $((u, \mu),(\hat{u}, \hat{\mu})) \in \mathcal{P}_{m}((x, y),(\hat{x}, \hat{y}))$ if there exist $\left(\left(x^{k}, y^{k}\right)\right)_{k=0}^{m}$ and $\left(\left(u^{k}, \mu^{k}\right)\right)_{k=0}^{m} m$-directed paths from $(x, y)$ to $(\hat{x}, \hat{y})$ and from $(u, \mu)$ to $(\hat{u}, \hat{\mu})$, respectively, such that $\left(\left(u^{k-1}, \mu^{k-1}\right),\left(u^{k}, \mu^{k}\right)\right) \in \mathcal{P}_{1}^{*}\left(\left(x^{k-1}, y^{k-1}\right),\left(x^{k}, y^{k}\right)\right)$ for $k=1, \ldots, m$. In particular, for all $(v, w) \in \mathcal{H}(x, y)$ such that

$$
(u(t), \mu(t))=\left(\int_{0}^{1} v(t, s) d s, \int_{0}^{1} w(t, s) d s\right)
$$

there exists $(\hat{v}, \hat{w}) \in \mathcal{H}(\hat{x}, \hat{y})$ such that, a.e. $s \in[0,1]$ and all $t \in[0,1]$,

$$
\begin{aligned}
& (\hat{u}(t), \hat{\mu}(t))=\left(\int_{0}^{1} \hat{v}(t, s) d s, \int_{0}^{1} \hat{w}(t, s) d s\right), \\
& \|(v(t, s), w(t, s))-(\hat{v}(t, s), \hat{w}(t, s))\| \leq \phi(t, s) p_{m}((x, y),(\hat{x}, \hat{y})),
\end{aligned}
$$


and

$$
0 \preceq \sigma_{*}(i)\left(\hat{v}_{i}(t, s)-v_{i}(t, s)\right) \quad \forall i=1, \ldots, N_{*} .
$$

$\left(\mathrm{P}_{m^{n}}\right)$ For $n \geq 2$ and $(\hat{x}, \hat{y}) \in[(x, y)]_{G}^{m^{n}}$, the property $\mathcal{P}_{m^{n}}^{*}((x, y),(\hat{x}, \hat{y}))$ is defined inductively. Hence, for $((u, \mu),(\hat{u}, \hat{\mu})) \in \mathcal{P}_{m^{n}}((x, y),(\hat{x}, \hat{y}))$, one has $(\hat{u}, \hat{\mu}) \in[(x, y)]_{G}^{m^{n+1}}$ and for all $(v, w) \in \mathcal{H}(x, y)$ such that

$$
(u(t), \mu(t))=\left(\int_{0}^{1} v(t, s) d s, \int_{0}^{1} w(t, s) d s\right)
$$

there exists $(\hat{v}, \hat{w}) \in \mathcal{H}(\hat{x}, \hat{y})$ such that, a.e. $s \in[0,1]$ and all $t \in[0,1]$,

$$
\begin{aligned}
& (\hat{u}(t), \mu \hat{(t)})=\left(\int_{0}^{1} \hat{v}(t, s) d s, \int_{0}^{1} \hat{w}(t, s) d s\right), \\
& \|(v(t, s), w(t, s))-(\hat{v}(t, s), \hat{w}(t, s))\| \leq \phi(t, s) p_{m^{n}}((x, y),(\hat{x}, \hat{y})),
\end{aligned}
$$

and

$$
0 \preceq \sigma_{*}(i)\left(\hat{v}_{i}(t, s)-v_{i}(t, s)\right) \quad \forall i=1, \ldots, N_{*} .
$$

It follows from (i) and (ii) that Assumptions (i) and (ii) of Theorem 2.8 are satisfied.

Finally, (iii) and (iv) imply that Condition (iii) of Theorem 2.8 is satisfied. Indeed, Let $\left\{\left(x_{n}, y_{n}\right)\right\}$ be such that $\left(x_{n}, y_{n}\right) \in F\left(x_{n-1}, y_{n-1}\right) \cap\left[\left(x_{n-1}, y_{n-1}\right)\right]_{G}^{m^{n}}$ and $\left(\left(x_{n}, y_{n}\right),\left(x_{n+1}, y_{n+1}\right)\right) \in$ $\mathcal{P}_{m^{n}}\left(\left(x_{n-1}, y_{n-1}\right),\left(x_{n}, y_{n}\right)\right)$ for all $n \in \mathbb{N}$ and

$$
\sum_{n=1}^{\infty} p_{m^{n+1}}\left(\left(x_{n}, y_{n}\right),\left(x_{n+1}, y_{n+1}\right)\right)<\infty
$$

So, there exist $(x, y) \in C([0,1], E)$ and $(v, w)$ such that $\left\|\left(x_{n}, y_{n}\right)-(x, y)\right\|_{0} \rightarrow 0$, and a.e. $s \in[0,1]$ for all $t \in[0,1],\left\|(v(t, \cdot), w(t, \cdot))-\left(v_{n}(t, \cdot), w_{n}(t, \cdot)\right)\right\|_{1} \rightarrow 0,\left(v_{n}(t, s), w_{n}(t, s)\right) \rightarrow$ $(v(t, \cdot), w(t, \cdot))$ and, by (iii), $0 \preceq \sigma_{*}(i)\left(x_{i}(t, s)-x_{n, i}(t, s)\right)$ for all $i=1, \ldots, N_{*}$.

It follows from (ii) that, for every $n \in \mathbb{N}$, there exists $\left(\hat{v}_{n}, \hat{w}_{n}\right) \in \mathcal{H}\left(x, y_{n}\right)$ such that

$$
\left\|\left(v_{n}(t, s), w_{n}(t, s)\right)-\left(\hat{v}_{n}(t, s), \hat{w}_{n}(t, s)\right)\right\| \leq \phi(t, s)\left\|x_{n}-x\right\|_{0} \quad \text { a.e. } s \in[0,1], \forall t \in[0,1]
$$

Therefore,

$$
\left(\hat{v}_{n}(t, s), \hat{w}_{n}(t, s)\right) \rightarrow(v(t, s), w(t, s)) \quad \text { a.e. } s \in[0,1], \forall t \in[0,1]
$$

Assumption (iv) implies that $(v, w) \in \mathcal{H}(x, y)$, and hence $(x, y) \in F(x, y)$.

Finally, Theorem 2.8 gives the conclusion.

\section{Examples}

In this section, we present some examples to illustrate the previous theorems. For the sake of simplicity, most of them will be given for systems of Hammerstein integral equations and continuous functions. 
Example 7.1 Let $E_{1}$ and $E_{2}$ be two Banach spaces endowed with a partial order. For $i=1,2$, let $g_{i}:[0,1] \times[0,1] \rightarrow[0, \infty)$ be continuous maps such that

$$
\sup _{t \in[0,1]}\left\|g_{1}(t, \cdot)+g_{2}(t, \cdot)\right\|_{1}<1
$$

For $i, j=1,2$, let $a_{i, j}:[0,1] \times E_{j} \rightarrow E_{i}$ be continuous functions satisfying the following condition:

(a) For $j=1,2$ and every $u, \hat{u} \in C\left([0,1], E_{j}\right)$ such that $u(s) \preceq \hat{u}(s)$ for all $s \in[0,1]$, one has

$$
\begin{aligned}
& a_{i, j}(s, u(s)) \preceq a_{i, j}(s, \hat{u}(s)) \quad \text { and } \\
& \left\|a_{i, j}(s, \hat{u}(s))-a_{i, j}(s, u(s))\right\| \leq\|\hat{u}-u\|_{0} \quad \forall s \in[0,1], \forall i=1,2 .
\end{aligned}
$$

We consider the system of Hammerstein integral equations:

$$
\left\{\begin{array}{l}
u(t)=\int_{0}^{1} g_{1}(t, s)\left(a_{1,1}(s, u(s))-a_{1,2}(s, v(s))\right) d s \\
v(t)=\int_{0}^{1} g_{2}(t, s)\left(-a_{2,1}(s, u(s))+a_{2,2}(s, v(s))\right) d s, \quad \forall t \in[0,1] .
\end{array}\right.
$$

If there exists $\left(u_{0}, v_{0}\right) \in C\left([0,1], E_{1} \times E_{2}\right)$ such that

$$
\left\{\begin{array}{l}
u_{0}(t) \preceq \int_{0}^{1} g_{1}(t, s)\left(a_{1,1}\left(s, u_{0}(s)\right)-a_{1,2}\left(s, v_{0}(s)\right)\right) d s, \\
v_{0}(t) \succeq \int_{0}^{1} g_{2}(t, s)\left(-a_{2,1}\left(s, u_{0}(s)\right)+a_{2,2}\left(s, v_{0}(s)\right)\right) d s, \quad \forall t \in[0,1],
\end{array}\right.
$$

then Theorem 5.1 implies that there exists $\left(u^{*}, v^{*}\right) \in C\left([0,1], E_{1} \times E_{2}\right)$ a solution of (7.1). Indeed, fix $\sigma(1)=1$ and $\sigma(2)=-1$. Let $(u, v),(\hat{u}, \hat{v}) \in C\left([0,1], E_{1} \times E_{2}\right)$ be such that

$$
0 \preceq \sigma(1)(\hat{u}(s)-u(s)) \quad \text { and } \quad 0 \preceq \sigma(2)(\hat{v}(s)-v(s)) \quad \forall s \in[0,1]
$$

that is

$$
u(s) \preceq \hat{u}(s) \quad \text { and } \quad \hat{v}(s) \preceq v(s) \quad \forall s \in[0,1] .
$$

Let us denote for $i=1,2$,

$$
\begin{aligned}
& w_{i}(t, s)=(-1)^{i+1} g_{i}(t, s)\left(a_{i, 1}(s, u(s))-a_{i, 2}(s, v(s))\right), \\
& \hat{w}_{i}(t, s)=(-1)^{i+1} g_{i}(t, s)\left(a_{i, 1}(s, \hat{u}(s))-a_{i, 2}(s, \hat{v}(s))\right) .
\end{aligned}
$$

It follows from (a) that

$$
w_{1}(t, s) \preceq \hat{w}_{1}(t, s), \quad \hat{w}_{2}(t, s) \preceq w_{2}(t, s),
$$

and

$$
\left\|w_{i}(t, s)-\hat{w}_{i}(t, s)\right\| \leq g_{i}(t, s)\left(\|\hat{u}-u\|_{0}+\|\hat{v}-v\|_{0}\right) \quad \forall t, s \in[0,1]
$$

So,

$$
0 \preceq \sigma(i)\left(\hat{w}_{i}(t, s)-w_{i}(t, s)\right) \quad \forall t, s \in[0,1], \forall i=1,2 ;
$$


and

$$
\begin{aligned}
& \left\|\left(w_{1}(t, s), w_{2}(t, s)\right)-\left(\hat{w}_{1}(t, s), \hat{w}_{2}(t, s)\right)\right\| \\
& \quad \leq\left(g_{1}(t, s)+g_{2}(t, s)\right)\|(u, v)-(\hat{u}, \hat{v})\|_{0} \quad \forall t, s \in[0,1] .
\end{aligned}
$$

Thus, Assumption (ii) of Theorem 5.1 is satisfied. Assumption (i) follows directly from (7.2), and (iii) is trivially satisfied.

Observe that if one defines inductively

$$
\left\{\begin{array}{l}
u_{n}(t, s)=\int_{0}^{1} g_{1}(t, s)\left(a_{1,1}\left(s, u_{n-1}(s)\right)-a_{1,2}\left(s, v_{n-1}(s)\right)\right) d s \\
v_{n}(t, s)=\int_{0}^{1} g_{2}(t, s)\left(-a_{2,1}\left(s, u_{n-1}(s)\right)+a_{2,2}\left(s, v_{n-1}(s)\right)\right) d s
\end{array}\right.
$$

then $\left\{\left(\sigma(1) u_{n}, \sigma(2) v_{n}\right)\right\}$ is a nondecreasing sequence converging to $\left(\sigma(1) u^{*}, \sigma(2) v^{*}\right)$, where $\left(u^{*}, v^{*}\right)$ is a solution of (7.1).

In the following example, we reverse the signs in the right member of (7.1).

Example 7.2 For $i, j=1,2$, let $E_{i}, g_{i}$ and $a_{i, j}$ be as in Example 7.1. We consider the system of Hammerstein integral equations:

$$
\left\{\begin{array}{l}
u(t)=\int_{0}^{1} g_{1}(t, s)\left(-a_{1,1}(s, u(s))+a_{1,2}(s, v(s))\right) d s, \\
v(t)=\int_{0}^{1} g_{2}(t, s)\left(a_{2,1}(s, u(s))-a_{2,2}(s, v(s))\right) d s, \quad \forall t \in[0,1] .
\end{array}\right.
$$

If there exists $\left(u_{0}, v_{0}\right) \in C\left([0,1], E_{1} \times E_{2}\right)$ such that

$$
\left\{\begin{array}{l}
u_{0}(t) \succeq \int_{0}^{1} g_{1}(t, s)\left(-a_{1,1}\left(s, u_{0}(s)\right)+a_{1,2}\left(s, v_{0}(s)\right)\right) d s, \\
v_{0}(t) \preceq \int_{0}^{1} g_{2}(t, s)\left(a_{2,1}\left(s, u_{0}(s)\right)-a_{2,2}\left(s, v_{0}(s)\right)\right) d s, \quad \forall t \in[0,1]
\end{array}\right.
$$

then Theorem 4.1 implies that there exists $\left(u^{*}, v^{*}\right) \in C\left([0,1], E_{1} \times E_{2}\right)$ a solution of (7.3). The proof is analogous to the one given in the previous example by taking $\sigma(1)=-1$ and $\sigma(2)=1$. Observe that if we define inductively

$$
\left\{\begin{array}{l}
u_{n}(t, s)=\int_{0}^{1} g_{1}(t, s)\left(-a_{1,1}\left(s, u_{n-1}(s)\right)+a_{1,2}\left(s, v_{n-1}(s)\right)\right) d s \\
v_{n}(t, s)=\int_{0}^{1} g_{2}(t, s)\left(a_{2,1}\left(s, u_{n-1}(s)\right)-a_{2,2}\left(s, v_{n-1}(s)\right)\right) d s
\end{array}\right.
$$

the sequence $\left\{\left(u_{n}, v_{n}\right)\right\}$ converges to $\left(u^{*}, v^{*}\right)$. However, it is not monotone. In fact,

$$
\begin{array}{ll}
u_{n} \succeq u_{n+1} \quad \text { and } & v_{n} \preceq v_{n+1} \quad \text { if } n \text { is even, } \\
u_{n} \preceq u_{n+1} \quad \text { and } & v_{n} \succeq v_{n+1} \quad \text { if } n \text { is odd. }
\end{array}
$$

We can also consider other signs in the right member of (7.1).

Example 7.3 For $i, j=1,2$, let $E_{i}, g_{i}$ and $a_{i, j}$ be as in Example 7.1. We consider the system of Hammerstein integral equations:

$$
\left\{\begin{array}{l}
u(t)=\int_{0}^{1} g_{1}(t, s)\left(\theta_{1,1} a_{1,1}(s, u(s))+\theta_{1,2} a_{1,2}(s, v(s))\right) d s, \\
v(t)=\int_{0}^{1} g_{2}(t, s)\left(\theta_{2,1} a_{2,1}(s, u(s))+\theta_{2,2} a_{2,2}(s, v(s))\right) d s, \quad \forall t \in[0,1],
\end{array}\right.
$$


where $\theta_{i, j} \in\{-1,1\}$ for $i, j=1,2$. We assume that there exist $\sigma_{0}(1), \sigma_{0}(2) \in\{-1,1\}$ and $\left(u_{0}, v_{0}\right) \in C\left([0,1], E_{1} \times E_{2}\right)$ such that, for all $t \in[0,1]$,

$$
\left\{\begin{array}{l}
0 \leq \sigma_{0}(1)\left(\int_{0}^{1} g_{1}(t, s)\left(\theta_{1,1} a_{1,1}\left(s, u_{0}(s)\right)+\theta_{1,2} a_{1,2}\left(s, v_{0}(s)\right)\right) d s-u_{0}(t)\right) \\
0 \leq \sigma_{0}(2)\left(\int_{0}^{1} g_{2}(t, s)\left(\theta_{2,1} a_{2,1}\left(s, u_{0}(s)\right)+\theta_{2,2} a_{2,2}\left(s, v_{0}(s)\right)\right) d s-v_{0}(t)\right) .
\end{array}\right.
$$

It follows from Theorem 3.1 that there exists $\left(u^{*}, v^{*}\right) \in C\left([0,1], E_{1} \times E_{2}\right)$ a solution of (7.3). Indeed, let

$$
\sigma_{i}(j)=\theta_{i, j} \quad \text { for } i, j=1,2
$$

For $(u, v),(\hat{u}, \widetilde{v}) \in C\left([0,1], E_{1} \times E_{2}\right)$ such that

$$
u(s) \preceq \hat{u}(s) \quad \text { and } \quad v(s) \preceq \widetilde{v}(s) \quad \forall s \in[0,1]
$$

let us denote, for $i=1,2$,

$$
\begin{aligned}
& w_{i}(t, s)=g_{i}(t, s)\left(\theta_{i, 1} a_{i, 1}(s, u(s))+\theta_{i, 2} a_{i, 2}(s, v(s))\right), \\
& \widehat{w}_{i}(t, s)=g_{i}(t, s)\left(\theta_{i, 1} a_{i, 1}(s, \hat{u}(s))+\theta_{i, 2} a_{i, 2}(s, v(s))\right), \\
& \widetilde{w}_{i}(t, s)=g_{i}(t, s)\left(\theta_{i, 1} a_{i, 1}(s, u(s))+\theta_{i, 2} a_{i, 2}(s, \widetilde{v}(s))\right) .
\end{aligned}
$$

It follows from Condition (a) in Example 7.1 that

$$
0 \preceq \sigma_{i}(1)\left(\widehat{w}_{i}(t, s)-w_{i}(t, s)\right), \quad 0 \preceq \sigma_{i}(2)\left(\widetilde{w}_{i}(t, s)-w_{i}(t, s)\right),
$$

and

$$
\begin{aligned}
\left\|w_{i}(t, s)-\widehat{w}_{i}(t, s)\right\| & \leq g_{i}(t, s)\|\hat{u}-u\|_{0}, \\
\left\|w_{i}(t, s)-\widetilde{w}_{i}(t, s)\right\| & \leq g_{i}(t, s)\|\widetilde{v}-v\|_{0}, \quad \forall t, s \in[0,1] .
\end{aligned}
$$

So, Assumption (ii) of Theorem 3.1 is satisfied. Assumption (i) follows directly from (7.6), and (iii) is trivially satisfied.

Observe that if we define inductively

$$
\left\{\begin{array}{l}
u_{n}(t, s)=\int_{0}^{1} g_{1}(t, s)\left(\theta_{1,1} a_{1,1}\left(s, u_{n-1}(s)\right)+\theta_{1,2} a_{1,2}\left(s, v_{n-1}(s)\right)\right) d s, \\
v_{n}(t, s)=\int_{0}^{1} g_{2}(t, s)\left(\theta_{2,1} a_{2,1}\left(s, u_{n-1}(s)\right)+\theta_{2,2} a_{2,2}\left(s, v_{n-1}(s)\right)\right) d s,
\end{array}\right.
$$

the sequence $\left\{\left(u_{n}, v_{n}\right)\right\}$ converges to $\left(u^{*}, v^{*}\right)$. However, it is not monotone. Moreover, we can have

$$
\begin{aligned}
& u_{n} \npreceq u_{n+1} \quad \text { and } \quad u_{n} \nsucceq u_{n+1} \quad \forall n \in \mathbb{N}, \\
& v_{n} \npreceq v_{n+1} \quad \text { and } \quad v_{n} \nsucceq v_{n+1} \quad \forall n \in \mathbb{N} .
\end{aligned}
$$

Here is an example where the contraction condition may not be satisfied between every pairs of elements. 
Example 7.4 Let us consider the system of Hammerstein integral equations

$$
u(t)=\int_{0}^{1} h(t, s, u(s)) d s, \quad \forall t \in[0,1]
$$

where $h:[0,1] \times[0,1] \times \mathbb{R}^{4} \rightarrow \mathbb{R}^{4}$ is given by

$$
h(t, s, u)=\left(\begin{array}{l}
h_{1}(t, s, u) \\
h_{2}(t, s, u) \\
h_{3}(t, s, u) \\
h_{4}(t, s, u)
\end{array}\right)=\left(\begin{array}{l}
a_{1,1}\left(t, s, u_{1}, u_{2}\right)-b_{1,1}\left(t, s, u_{3}, u_{4}\right) \\
a_{1,2}\left(t, s, u_{1}, u_{2}\right)-b_{1,2}\left(t, s, u_{3}, u_{4}\right) \\
a_{2,1}\left(t, s, u_{1}, u_{2}\right)+b_{2,1}\left(t, s, u_{3}, u_{4}\right) \\
a_{2,2}\left(t, s, u_{1}, u_{2}\right)+b_{2,2}\left(t, s, u_{3}, u_{4}\right)
\end{array}\right) \text {, }
$$

with $a_{i, j}, b_{i, j}$ continuous functions satisfying the following conditions:

(a) There exists $c_{1}, c_{2} \in(0,1]$ such that, for every $\left(x_{1}, x_{2}\right),\left(\hat{x}_{1}, \hat{x}_{2}\right) \in \mathbb{R}^{2}$ with $x_{1} \leq \hat{x}_{1}$ and $x_{2} \leq \hat{x}_{2}$, one has, for $i=1,2$ and for all $t, s \in[0,1]$,

$$
\begin{aligned}
0 & \leq a_{i, 2}\left(t, s, \hat{x}_{1}, \hat{x}_{2}\right)-a_{i, 2}\left(t, s, x_{1}, x_{2}\right) \\
& \leq c_{i}\left(a_{i, 1}\left(t, s, \hat{x}_{1}, \hat{x}_{2}\right)-a_{i, 1}\left(t, s, x_{1}, x_{2}\right)\right), \\
0 & \leq b_{i, 2}\left(t, s, \hat{x}_{1}, \hat{x}_{2}\right)-b_{i, 2}\left(t, s, x_{1}, x_{2}\right) \\
& \leq c_{i}\left(b_{i, 1}\left(t, s, \hat{x}_{1}, \hat{x}_{2}\right)-b_{i, 1}\left(t, s, x_{1}, x_{2}\right)\right) .
\end{aligned}
$$

(b) For $i=1,2$, there exist $\alpha_{i, 1}, \alpha_{i, 2}, \beta_{i, 1}, \beta_{i, 2}:[0,1] \times[0,1] \rightarrow[0, \infty)$ continuous such that

$$
\begin{aligned}
& \sup _{t \in[0,1]}\left\|\sum_{i=1}^{2} \alpha_{i, 1}(t, \cdot)+c_{i} \alpha_{i, 2}(t, \cdot)\right\|_{1}<1, \\
& \sup _{t \in[0,1]}\left\|\sum_{i=1}^{2} \beta_{i, 1}(t, \cdot)+c_{i} \beta_{i, 2}(t, \cdot)\right\|_{1}<1,
\end{aligned}
$$

and, for every $\left(x_{1}, x_{2}\right),\left(\hat{x}_{1}, \hat{x}_{2}\right) \in \mathbb{R}^{2}$ such that $x_{1} \leq \hat{x}_{1}$ and $x_{2} \leq \hat{x}_{2}$, one has for all $t, s \in[0,1]$

$$
\begin{aligned}
& a_{i, 1}\left(t, s, \hat{x}, \hat{x}_{2}\right)-a_{i, 1}\left(t, s, x_{1}, x_{2}\right) \leq \alpha_{i, 1}(t, s)\left(\hat{x}_{1}-x_{1}\right)+\alpha_{i, 2}(t, s)\left(\hat{x}_{2}-x_{2}\right), \\
& b_{i, 1}\left(t, s, \hat{x}, \hat{x}_{2}\right)-b_{i, 1}\left(t, s, x_{1}, x_{2}\right) \leq \beta_{i, 1}(t, s)\left(\hat{x}_{1}-x_{1}\right)+\beta_{i, 2}(t, s)\left(\hat{x}_{2}-x_{2}\right) .
\end{aligned}
$$

(c) There exists $\theta_{1}, \theta_{2} \in\{-1,1\}$ such that

$$
\begin{aligned}
& 0 \leq \theta_{1} \int_{0}^{1} h_{2}(t, s, 0,0) d s \leq \theta_{1} c_{1} \int_{0}^{1} h_{1}(t, s, 0,0) \\
& 0 \leq \theta_{2} \int_{0}^{1} h_{4}(t, s, 0,0) d s \leq \theta_{2} c_{2} \int_{0}^{1} h_{3}(t, s, 0,0) \quad \forall t \in[0,1] .
\end{aligned}
$$

Then Corollary 3.4 implies that there exists $u^{*} \in C\left([0,1], \mathbb{R}^{4}\right)$, a solution of (7.7). Indeed, for $i=1,2$, let $E_{i}=\mathbb{R}^{2}$ endowed with the norm $\left\|\left(x_{1}, x_{2}\right)\right\|=\max \left\{\left|x_{1}\right|,\left|x_{2}\right|\right\}$ and the partial order

$$
\left(x_{1}, x_{2}\right) \preceq_{i}\left(\hat{x_{1}}, \hat{x_{2}}\right) \quad \Longleftrightarrow 0 \leq \hat{x}_{2}-x_{2} \leq c_{i}\left(\hat{x}_{1}-x_{1}\right) .
$$


It follows from (a) that, for all $x=\left(x_{1}, x_{2}\right) \preceq_{1} \hat{x}=\left(\hat{x}_{1}, \hat{x_{2}}\right)$ and for all $y=\left(y_{1}, y_{2}\right) \in \mathbb{R}^{2}$,

$$
\begin{aligned}
& 0 \leq h_{2}(t, s, \hat{x}, y)-h_{2}(t, s, x, y) \leq c_{1}\left(h_{1}(t, s, \hat{x}, y)-h_{1}(t, s, x, y)\right) \\
& 0 \leq h_{4}(t, s, \hat{x}, y)-h_{4}(t, s, x, y) \leq c_{2}\left(h_{3}(t, s, \hat{x}, y)-h_{3}(t, s, x, y)\right) \quad \forall t, s \in[0,1] .
\end{aligned}
$$

So,

$$
\begin{aligned}
& \left(h_{1}(t, s, x, y), h_{2}(t, s, x, y)\right) \preceq_{1}\left(h_{1}(t, s, \hat{x}, y), h_{2}(t, s, \hat{x}, y)\right), \\
& \left(h_{3}(t, s, x, y), h_{4}(t, s, x, y)\right) \preceq_{2}\left(h_{3}(t, s, \hat{x}, y), h_{4}(t, s, \hat{x}, y)\right), \quad \forall t, s \in[0,1] .
\end{aligned}
$$

Similarly, for all $x \in \mathbb{R}^{2}$ and all $y \preceq_{2} \hat{y}$, one has

$$
\begin{aligned}
& \left(h_{1}(t, s, x, \hat{y}), h_{2}(t, s, x, \hat{y})\right) \preceq_{1}\left(h_{1}(t, s, x, y), h_{2}(t, s, x, y)\right), \\
& \left(h_{3}(t, s, x, y), h_{4}(t, s, x, y)\right) \preceq_{2}\left(h_{3}(t, s, x, \hat{y}), h_{4}(t, s, x, \hat{y})\right), \quad \forall t, s \in[0,1] .
\end{aligned}
$$

Thus, Assumption (iii) of Corollary 3.4 is satisfied.

Conditions (a) and (b) implies that, for all $y \in \mathbb{R}^{2}$ and all $x \preceq_{1} \hat{x}$, one has

$$
\begin{aligned}
& \left\|\left(h_{1}(t, s, \hat{x}, y), h_{2}(t, s, \hat{x}, y)\right)-\left(h_{1}(t, s, x, y), h_{2}(t, s, x, y)\right)\right\| \\
& \quad \leq a_{1,1}(t, s, \hat{x})-a_{1,1}(t, s, x) \\
& \quad \leq \alpha_{1,1}(t, s)\left(\hat{x}_{1}-x_{1}\right)+\alpha_{1,2}(t, s)\left(\hat{x}_{2}-x_{2}\right) \\
& \quad \leq\left(\alpha_{1,1}(t, s)+c_{1} \alpha_{1,2}(t, s)\right)\|\hat{x}-x\| .
\end{aligned}
$$

Similarly, for all $x \preceq_{1} \hat{x}$ and all $y \preceq_{2} \hat{y}$, one has

$$
\begin{aligned}
& \left\|\left(h_{1}(t, s, x, \hat{y}), h_{2}(t, s, x, \hat{y})\right)-\left(h_{1}(t, s, x, y), h_{2}(t, s, x, y)\right)\right\| \\
& \quad \leq\left(\beta_{1,1}(t, s)+c_{1} \beta_{1,2}(t, s)\right)\|\hat{y}-y\|, \\
& \left\|\left(h_{3}(t, s, \hat{x}, y), h_{4}(t, s, \hat{x}, y)\right)-\left(h_{3}(t, s, x, y), h_{4}(t, s, x, y)\right)\right\| \\
& \quad \leq\left(\alpha_{2,1}(t, s)+c_{2} \alpha_{2,2}(t, s)\right)\|\hat{x}-x\|, \\
& \left\|\left(h_{3}(t, s, x, \hat{y}), h_{4}(t, s, x, \hat{y})\right)-\left(h_{3}(t, s, x, y), h_{4}(t, s, x, y)\right)\right\| \\
& \quad \leq\left(\beta_{2,1}(t, s)+c_{2} \beta_{2,2}(t, s)\right)\|\hat{y}-y\| .
\end{aligned}
$$

Therefore, Assumption (iv) of Corollary 3.4 is satisfied.

Condition (c) implies that

$$
\begin{aligned}
&(0,0) \preceq_{1}\left(\int_{0}^{1} h_{1}(t, s, 0,0) d s, \int_{0}^{1} h_{2}(t, s, 0,0) d s\right) \\
&\left(\operatorname{or}\left(\int_{0}^{1} h_{1}(t, s, 0,0) d s, \int_{0}^{1} h_{2}(t, s, 0,0) d s\right) \preceq_{1}(0,0)\right), \\
&(0,0) \preceq_{2}\left(\int_{0}^{1} h_{3}(t, s, 0,0) d s, \int_{0}^{1} h_{4}(t, s, 0,0) d s\right)
\end{aligned}
$$




$$
\left(\text { or }\left(\int_{0}^{1} h_{3}(t, s, 0,0) d s, \int_{0}^{1} h_{4}(t, s, 0,0) d s\right) \preceq_{2}(0,0)\right) \text {. }
$$

This ensures that Assumption (ii) is satisfied. Finally, it is clear that Assumptions (i) and (v) hold. So, Corollary 3.4 implies the existence of $u^{*} \in C\left([0,1], \mathbb{R}^{4}\right)$ a solution of (7.7).

Here is an example of a system of Hammerstein integral inclusions.

Example 7.5 Consider the system of Hammerstein integral inclusions:

$$
\left\{\begin{array}{l}
x(t) \in \int_{0}^{1} H_{1}(t, s, x(s), y(s)) d s, \\
y(t) \in \int_{0}^{1} H_{2}(t, s, x(s), y(s)) d s, \quad \forall t \in[0,1],
\end{array}\right.
$$

where

$$
\begin{aligned}
& H_{1}(t, s, x, y)=a(x) b(y)+(t s)^{2}\left[\frac{1-y}{2}, e^{-y}\right] ; \\
& H_{2}(t, s, x, y)=c(t, s, y)+ \begin{cases}{\left[\frac{1}{x-1}, 2-e^{x / 8}\right]} & \text { if } x<0, \\
{\left[-(x+3)^{2}, e^{-x / 8}\right]} & \text { if } x \geq 0 ;\end{cases} \\
& a(x)= \begin{cases}1-\frac{1}{x+2} & \text { if } x \geq 0 \\
\frac{1}{2-x} & \text { if } x<0 ;\end{cases} \\
& b(y)= \begin{cases}\frac{1}{y+3} & \text { if } y \geq 0, \\
1-\frac{2}{3-y} & \text { if } y<0\end{cases} \\
& c(t, s, y)=\frac{(t+1)(2 y-5)}{16 \sqrt{s}} .
\end{aligned}
$$

For $(x, y),(\hat{x}, \hat{y}) \in \mathbb{R}^{2}$ such that $x \leq \hat{x}$ and $y \geq \hat{y}$, and for $u \in H_{1}(t, s, x, y), v \in H_{2}(t, s, x, y)$, one has

$$
\begin{aligned}
& u=a(x) b(y)+(t s)^{2} w \quad \text { for some } w \in\left[\frac{1-y}{2}, e^{-y}\right], \\
& v=c(t, s, y)+z \text { for some } z \in \begin{cases}{\left[\frac{1}{x-1}, 2-e^{x / 8}\right]} & \text { if } x<0, \\
{\left[-(x+3)^{2}, e^{-x / 8}\right]} & \text { if } x \geq 0 .\end{cases}
\end{aligned}
$$

Let

$$
\begin{aligned}
& \hat{u}=a(\hat{x}) b(\hat{y})+(t s)^{2} \max \left\{w, \frac{1-\hat{y}}{2}\right\}, \\
& \hat{v}=c(t, s, \hat{y})+ \begin{cases}\min \left\{z, 2-e^{\hat{x} / 8}\right\}, & \text { if } \hat{x}<0, \\
\min \left\{z, e^{-\hat{x} / 8}\right\} & \text { if } \hat{x} \geq 0 .\end{cases}
\end{aligned}
$$

Observe that

$$
(\hat{u}, \hat{v}) \in\left(H_{1}(t, s, \hat{x}, \hat{y}), H_{2}(t, s, \hat{x}, \hat{y})\right), \quad u \leq \hat{u} \text { and } \hat{v} \leq v .
$$


Also,

$$
\begin{aligned}
|\hat{u}-u| & \leq \frac{1}{4}|\hat{x}-x|+\left(\frac{2}{9}+\frac{(t s)^{2}}{2}\right)|\hat{y}-y| \\
& \leq\left(\frac{1}{4}+\frac{(t s)^{2}}{2}\right)(|\hat{x}-x|+|\hat{y}-y|), \\
|\hat{v}-v| & \leq \frac{1}{8}|\hat{x}-x|+\frac{2(t+1)}{16 \sqrt{s}}|\hat{y}-y| \leq \frac{(t+1)}{8 \sqrt{s}}(|\hat{x}-x|+|\hat{y}-y|) .
\end{aligned}
$$

So,

$$
\|(\hat{u}, \hat{v})-(u, v)\| \leq \phi(t, s)\|(\hat{x}, \hat{y})-(x, y)\|,
$$

with

$$
\phi(t, s)=\frac{1}{4}+\frac{(t s)^{2}}{2}+\frac{(t+1)}{8 \sqrt{s}} .
$$

Observe that

$$
\sup _{t \in[0,1]}\|\phi(t, \cdot)\|_{1}<1
$$

Therefore, $\left(H_{1}, H_{2}\right)$ satisfies Assumption (ii) of Theorem 5.1 with $\sigma(1)=1$ and $\sigma(2)=-1$.

Let us take $\left(x_{0}, y_{0}\right)=(0,0)$ and

$$
\left(u_{0}(t, s), v_{0}(t, s)\right)=\left(\frac{1}{6}+(t s)^{2}, \frac{-5(t+1)}{16 \sqrt{s}}\right) \in\left(H_{1}(t, s, 0,0), H_{2}(t, s, 0,0)\right) .
$$

One has

$$
0 \leq \int_{0}^{1} u_{0}(t, s) d s \quad \text { and } \quad 0 \geq \int_{0}^{1} v_{0}(t, s) d s \quad \forall t \in[0,1] .
$$

So, Assumption (i) of Theorem 5.1 is satisfied. It is easy to see that $\left(H_{1}, H_{2}\right)$ satisfies Assumption (iii). Therefore, Theorem 5.1 ensures that (7.8) has a solution.

\section{Competing interests}

The authors declare that they have no competing interests.

Authors' contributions

All authors contributed equally to the writing of this paper. All authors read and approved the final manuscript.

\section{Acknowledgements}

The second author was partially supported by NSERC Canada.

Received: 15 April 2015 Accepted: 10 September 2015 Published online: 26 September 2015

\section{References}

1. Couchouron, J-F, Precup, R: Existence principles for inclusions of Hammerstein type involving noncompact acyclic multivalued maps. Electron. J. Differ. Equ. 2002, 4 (2002)

2. O'Regan, D, Precup, R: Fixed point theorems for set-valued maps and existence principles for integral inclusions. J. Math. Anal. Appl. 245, 594-612 (2000)

3. O'Regan, D, Precup, R: Integrable solutions of Hammerstein integral inclusions in Banach spaces. Dyn. Contin. Discrete Impuls. Syst., Ser. A Math. Anal. 9, 165-176 (2002) 
4. Papageorgiou, N, Yannakakis, N: Hammerstein integral inclusions in Banach spaces. In: Integral and Integrodifferential Equations. Ser. Math. Anal. Appl., vol. 2, pp. 279-293. Gordon \& Breach, Amsterdam (2000)

5. Biles, DC, Robinson, MP, Spraker, JS: Fixed point approaches to the solution of integral inclusions. Topol. Methods Nonlinear Anal. 25, 297-311 (2005)

6. Petruşel, A: Integral inclusions. Fixed point approaches. Comment. Math. Prace Mat. 40, 147-158 (2000)

7. Hong, S, Qiu, Z: Fixed points of discontinuous multivalued operators in ordered spaces with applications. Fixed Point Theory Appl. 2010, Article ID 745769 (2010)

8. Ran, ACM, Reurings, MCB: A fixed point theorem in partially ordered sets and some applications to matrix equations. Proc. Am. Math. Soc. 132, 1435-1443 (2004)

9. Nieto, JJ, Rodríguez-López, R: Contractive mapping theorems in partially ordered sets and applications to ordinary differential equations. Order 22, 223-239 (2005)

10. Nieto, JJ, Rodríguez-López, R: Existence and uniqueness of fixed point in partially ordered sets and applications to ordinary differential equations. Acta Math. Sin. Engl. Ser. 23, 2205-2212 (2007)

11. Jachymski, J: The contraction principle for mappings on a metric space with a graph. Proc. Am. Math. Soc. 136, 1359-1373 (2008)

12. Dinevari, T, Frigon, M: Fixed point results for multivalued contractions on a metric space with a graph. J. Math. Anal. Appl. 405, 507-517 (2013)

13. Opoǐcev, Vl: Heterogeneous and combined concave operators. Sib. Mat. Zh. 16(4), 597-605 (1975) (in Russian)

14. Opoicev, VI: Generalization of the theory of monotone and concave operators. Tr. Mosk. Mat. Obŝ. 36, 237-273, 295 (1978) (in Russian)

15. Guo, DJ, Lakshmikantham, V: Coupled fixed points of nonlinear operators with applications. Nonlinear Anal. 11, 623-632 (1987)

16. Gnana Bhaskar, T, Lakshmikantham, V: Fixed point theorems in partially ordered metric spaces and applications. Nonlinear Anal. 65, 1379-1393 (2006)

17. Hong, S: Fixed points for mixed monotone multivalued operators in Banach spaces with applications. J. Math. Anal. Appl. 337, 333-342 (2008)

\section{Submit your manuscript to a SpringerOpen ${ }^{\circ}$ journal and benefit from:}

- Convenient online submission

Rigorous peer review

- Immediate publication on acceptance

- Open access: articles freely available online

- High visibility within the field

- Retaining the copyright to your article 